\title{
How Emotional Arousal Enhances Episodic Memory
}

Link to publication record in Manchester Research Explorer

\section{Citation for published version (APA):}

Ack Baraly, K., Hot, P., Davidson, P., \& Talmi, D. (2016). How Emotional Arousal Enhances Episodic Memory. In J. H. Byrne (Ed.), Learning and Memory: A Comprehensive Reference (2nd ed.). Elsevier BV.

\section{Published in:}

Learning and Memory

\section{Citing this paper}

Please note that where the full-text provided on Manchester Research Explorer is the Author Accepted Manuscript or Proof version this may differ from the final Published version. If citing, it is advised that you check and use the publisher's definitive version.

\section{General rights}

Copyright and moral rights for the publications made accessible in the Research Explorer are retained by the authors and/or other copyright owners and it is a condition of accessing publications that users recognise and abide by the legal requirements associated with these rights.

\section{Takedown policy}

If you believe that this document breaches copyright please refer to the University of Manchester's Takedown Procedures [http://man.ac.uk/04Y6Bo] or contact uml.scholarlycommunications@manchester.ac.uk providing relevant details, so we can investigate your claim.

\section{open $\partial_{\text {ACCESS }}$}


How Emotional Arousal Enhances Episodic Memory

Kylee T. Ack Baraly

School of Psychology, University of Ottawa, and CNRS, LPNC UMR 5105, F-73000, Université Savoie Mont Blanc, Chambéry, France

kylee.ackbaraly@uottawa.ca

Pascal Hot

CNRS, LPNC UMR 5105, F-73000, Université Savoie Mont Blanc, Chambéry, France pascal.hot@univ-smb.fr

Patrick S. R. Davidson

School of Psychology, University of Ottawa

patrick.davidson@uottawa.ca

Deborah Talmi

School of Psychological Sciences, University of Manchester, and Princeton Neuroscience Institute, Princeton University.

deborah.talmi@manchester.ac.uk 


\section{Table of Contents}

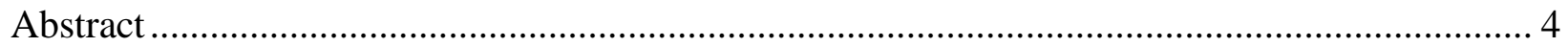

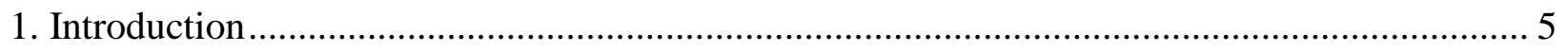

1.1 Emotion and Memory in Humans and Animals .......................................................... 5

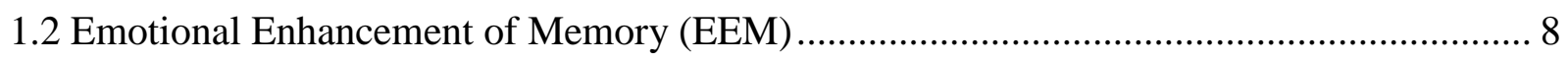

2. Persistent Emotional Memories .................................................................................. 9

2.1 The Memory Advantage of Emotional Stimuli Grows With Time ................................ 9

2.2 Post-Encoding Arousal Induction Alters Delayed Memory ........................................ 11

2.3 Central Role of the Amygdala in Human EEM ......................................................... 13

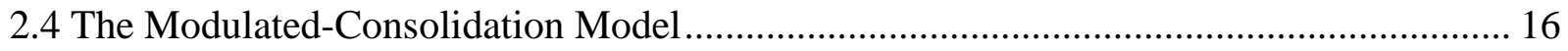

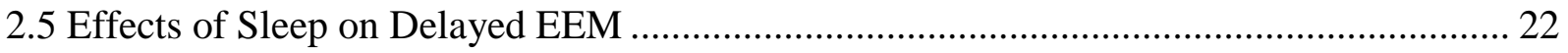

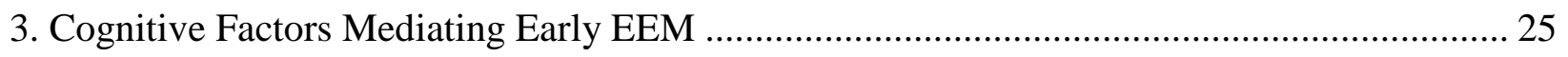

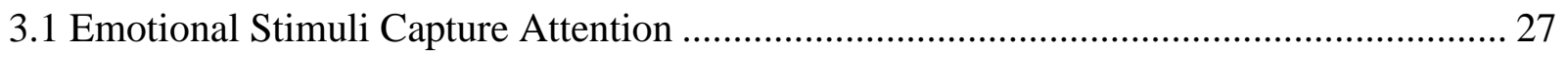

3.2 Emotional Stimuli are Semantically Inter-Related .............................................. 31

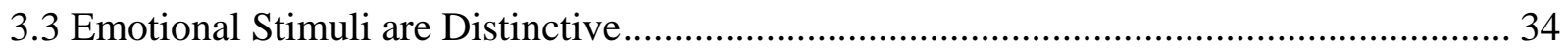

3.4 Joint Contribution of Cognitive Factors to Immediate EEM........................................ 35

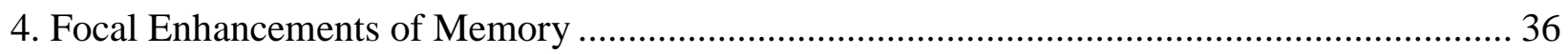

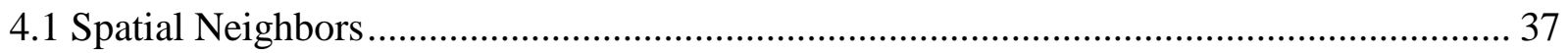

4.2 Temporal Neighbors: Anterograde and Retrograde Amnesia ..................................... 41

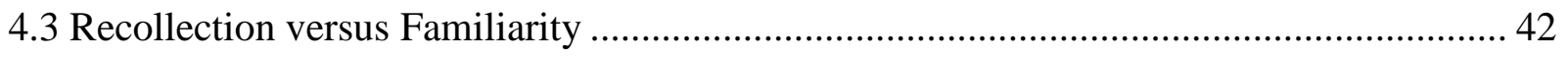

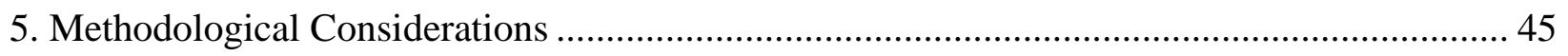

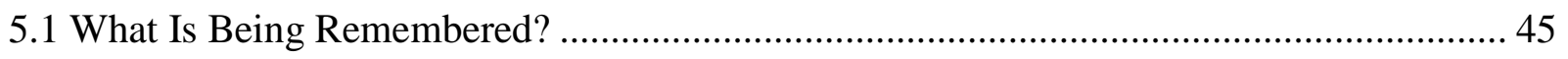

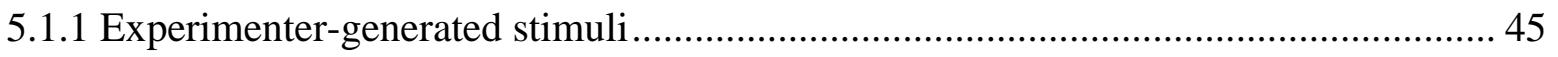

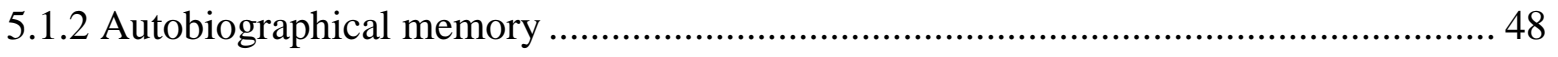

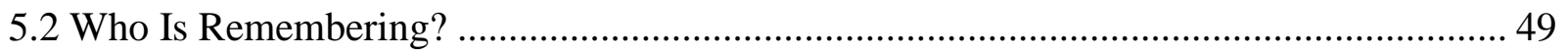




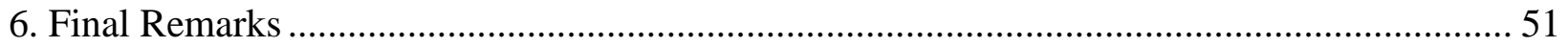

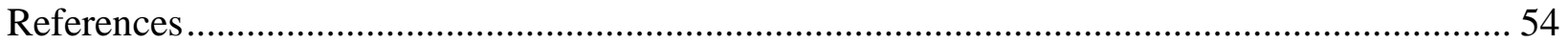




\begin{abstract}
50-100 words

This chapter reviews some key aspects of the relationship between emotion and episodic memory, focusing on the cognitive and neural systems that underlie the relationship. We differentiate between effects of emotion on early and late long-term memory, review evidence for the selectivity of these effects, and discuss existing models that best account for them. We reflect on and evaluate current practices in emotional memory research.

Keywords: arousal, attention, distinctiveness, emotion, episodic memory, long-term memory, modulation model, organization, recall, recognition, valence
\end{abstract}


"Of what significance the things you can forget? A little thought is sexton to all the world."

Henry David Thoreau

\section{Introduction}

Philosophers, politicians, and playwrights have long understood the interaction of emotion and cognition. Psychologists' interest has waxed and waned according to the zeitgeist, from early interest in the effects of motivation on perception inspired by psychodynamic ideas (The New Look) through denial of emotion and cognition as being worthy or tractable topics of inquiry (Radical Behaviorism) to a desire to study cognition in isolation from emotion (the Cognitive Revolution) to, finally, an understanding of the benefits of studying cognition, emotion, and the brain together (Cognitive and Affective Neuroscience).

This chapter reviews some key aspects of the relationship between emotion and episodic memory, focusing on the cognitive and neural systems that underlie the relationship. We aim to strike a balance between summarizing canonical findings, highlighting interesting new data and theories, and proposing useful questions for the future. Given the rapid growth in the field, our review is necessarily selective. In particular, for readers seeking in-depth coverage of moodcongruent memory and traumatic memory, Eich et al. (2008) provide a cogent and still highlyrelevant review.

\subsection{Emotion and Memory in Humans and Animals}

When people use the word 'memory,' it is often to describe the act of recollecting the details of a previous event, with the awareness of their having personally experienced it before. This colloquial understanding of episodic memory fits well with definitions from many researchers (Conway, 2009; James, 1890; Roediger et al., 2007; Tulving, 1985). Crucially, most researchers would accept that episodic memory is one of several kinds of memory, each with its own cognitive operating rules and neural bases (a 'memory systems' view). According to this predominant framework, episodic memory can be distinguished from semantic memory: Although both involve declarative mental content, episodic memory allows us to access the details of a previous experience to create conscious recollection from a first-person perspective. Episodic memories are encoded, stored, consolidated (and there may be more than one kind of consolidation, cf. Dudai, 2004) and retrieved. Emotion probably influences all of these stages. 
The way that episodic memory is conceptualized influences the way that it is studied. There are arguably many criteria on which episodic memory differs from other kinds of memory, but which of those criteria is most important will vary among researchers (Conway, 2009; Tulving, 1985). Some emphasize the first-person subjective sense of 'mental time travel' that accompanies the retrieval of an episodic memory (e.g., Wheeler et al., 1997). From this perspective, a participant verbally expressing a strong subjective sense of reliving or reexperiencing might be seen as the best index of episodic memory. In contrast, for those researchers who emphasize episodic memory's allowing an organism to link disparate pieces of an experience together to create one coherent memory, the primary indicator of episodic memory might entail the ability to remember links between two unrelated bits of information. For those who emphasize episodic memory's importance for situating a past experience within a particular time and place, the ability to recollect those contextual details may be key (for a variety of integrative perspectives, see Moscovitch et al., 2016; Squire and Wixted, 2016; Teyler and Rudy, 2007). These different perspectives go hand-in-hand with different theories of the brain bases of episodic memory, although most agree that the medial temporal lobe (in particular, the hippocampus) and prefrontal cortex are crucial.

Just as in the study of memory, but arguably even more so, the scientific community has found it difficult to agree on a definition for the term 'emotion' (Izard, 2010). Each theoretical affiliation in the study of emotion focuses on a subset of emotion phenomena, and that focus guides what research questions are thought to be interesting, what methodologies are used and, ultimately, how research findings are interpreted (Lazarus, 1991; for a historical review of emotion theories, see Barrett and Russell, 2015). One line of investigation concerns the origin of emotional responses. The notion that human emotion is linked to brain circuits that are conserved across mammals because they serve essential, shared survival tasks (LeDoux, 2012) has inspired burgeoning research (LeDoux, 2012; Panksepp, 2005). Similarly, much research is devoted to understanding how the reinforcement history of individuals results in emotional reactions to objectively neutral stimuli (Rolls, 2005). This work has not only illuminated the brain basis of emotion, but also yielded deep insights, for example by characterizing the distinction between threat and fear (LeDoux, 2014) or between wanting something, enjoying it, and learning about its value (Berridge et al., 2009), or by providing a mathematical understanding of emotional value 
learning (Schultz et al., 1997). At the other end of the spectrum, there is also much work on how our society and culture influence what we feel (Averill, 1980; Boiger and Mesquita, 2012).

Another broad line of investigation in emotion research is the distinction between different emotions. Discrete theories delineate the evolution, cognition and physiology of unique emotions such as disgust and anger (Ekman, 1992; Ekman et al., 1972; Izard, 2011; Panksepp, 1998) whereas other approaches focus on commonalities between emotions, from the dimensions that underlie them, most prevalently arousal and valence (Russell, 2003), to the components that they share (Ortony and Turner, 1990; Turner and Ortony, 1992), even conceiving emotions as constructed 'online' from domain-general psychological ingredients and brain networks (Barrett, 2014). The evaluation of the personal relevance of the environment is key to appraisal theories (Arnold, 1960; Lazarus, 1991; Roseman, 2011; Sander et al., 2005) and to psychological construction theory (Barrett and Russell, 2015), which place the extraction of meaning as central to the eventual emotional experience. Throughout this chapter, we will refer back to the theoretical conceptualization of emotion to suggest how those implicitly influence the way emotional memory is studied.

Throughout this chapter, we will make note of parallels between human and (non-human) animal findings. At the outset, we should note the obvious general complication that we must infer animals' psychological experience from non-verbal behavior. This is a particular challenge in episodic memory. Can non-humans have episodic memory in the same sense as humans do, or do some species merely have 'episodic-like memory'? Where (and how) does one draw the line (Pause et al., 2013)? These questions are difficult if not impossible to answer if one stresses the first-person subjective sense of 'mental time travel' as being the key indicator of episodic memory. Questions about episodic memory in animals become more tractable when one emphasizes the ability to bind disparate pieces of information together in memory and/or to remember precise spatial, temporal, or perceptual contextual details as being the crucial indicators of episodic memory.

Similarly, it is often remarked that it is difficult to understand inherently human experiences such as grief or elation by drawing on animal models. Not only do the feelings associated with emotion inaccessible in animals, but human emotions are also profoundly tuned to our own social-cognitive world. To an extent, the research on emotional memory has 
successfully avoided these issues by focusing on the dimensions of affective arousal and affective valence, where animal models have greater ecological validity. While that literature is not always explicit in its underlying assumptions about emotion, the interest in arousal and valence is logically consistent with Russell's dimensional model of core affect (Russell, 2003), which describes all emotional experiences as falling along a circle, defined across a twodimensional axis system comprising of valence (happy to unhappy) and arousal (calm to excited). In turn, the interest in arousal and valence has inevitably marginalized research questions that fit more naturally within other conceptualizations of emotion, such as the specific effects of discrete 'basic' emotions on memory (Chapman et al., 2013), or the role of cognitive factors (Section 3).

\subsection{Emotional Enhancement of Memory (EEM)}

Remembering past events is crucial for responding to present and future occurrences of similar events (Gershman and Daw, in press). Because the brain cannot maintain access to memories of all past experiences, it must prioritize those more likely to be useful. Reinforcement learning theories of emotion posit that our emotions provide an efficient summary of the overall value of stimuli and actions in service of optimal decision-making (e.g. Damasio et al., 1996); perhaps they have even evolved for that reason (Rolls, 2005). Therefore, the extent to which an event triggers affective arousal could indicate that greater resources should be dedicated to remembering it. For instance, you may not remember that your friend's dog can play catch, but you may recall that you were once intimidated when it growled at you, and that memory could help you avoid this dog in future.

Early research on arousal and memory is described in a number of previous reviews (Craik and Blankstein, 1975; Eysenck, 1976; Levonian, 1972; Matlin and Stang, 1978). One of the earliest views, referred to as the Yerkes-Dodson Law, posited that memory is boosted or impaired by arousal in an inverted-U relationship (Yerkes and Dodson, 1908). The YerkesDodson Law has been criticized because it is difficult to falsify (Neiss, 1988): Every finding (of impaired or enhanced memory) can be interpreted as falling on either end of the U-curve, without being able to independently predict where exactly on the curve one falls. Furthermore, indices of arousal do not correlate with each other; and memories for highly-arousing events (e.g., a near plane crash) can be recalled in great detail and last for many years, contrary to the 
prediction that extreme levels of arousal impair memory (McKinnon et al., 2015). Still, there is overwhelming evidence that moderate levels of arousal enhance memory (Kensinger, 2004; LaBar and Cabeza, 2006). Therefore, the prevalent paradigm in animals to study emotional memory is the one-trial avoidance learning paradigm, described in Section 2.4, where moderate shocks lead to greater avoidance of the punishing context than mild shocks (Gold and van Buskirk, 1978; Koriat et al., 2011). Similarly, much of the human work on the emotional enhancement of memory (EEM) explores the boundary conditions for the effects of affective arousal, for example, affective valence (Kensinger, 2004). In this chapter we will review how moderate levels of emotional arousal benefit memory, and the factors that influence its impact.

The human emotional memory literature has been particularly interested in the mechanism that allows emotional experiences to be remembered well long after they have occurred. This focus is often attributed to the famous quote from William James, that "[a]n impression may be so exciting emotionally as almost to leave a scar on the cerebral tissues" (James, 1890, p. 670). This interest is apparent, for example, in research into the good memory people have for significant public events (see Section 5.1.2). The focus of investigation on delayed emotional memory is also related to the finding that the EEM effect is often greater after a delay, and indeed sometimes not observed at all - or even reversed - when memory is tested immediately after encoding (Craik and Blankstein, 1975; Kleinsmith and Kaplan, 1963; Sharot and Phelps, 2004). It is also related to proposals that while immediate effects of emotion on memory appear to rely on generic cognitive factors (Section 3), delayed effects require special mechanisms. We begin our review therefore with a consideration of the persistent effect of emotion on tests of late long-term episodic memory (LTM), and turn to EEM in early LTM in the following section.

\section{Persistent Emotional Memories}

In this section we will review three core findings in the emotional memory literature. These findings have been interpreted as supporting a particular model of EEM, the modulatedconsolidation hypothesis, which we describe and evaluate in Section 2.4. We end this section with a discussion of the influence of sleep on delayed EEM.

\subsection{The Memory Advantage of Emotional Stimuli Grows With Time}


Many researchers have demonstrated large EEM in tests that take place hours and days after encoding. Beginning with Kleinsmith and Kaplan $(1964,1963)$, recall of high-arousal paired associates was lowest on an immediate test ( 2 min after encoding), much higher after 45 minutes or one day, and even higher again when tested after one week (Kleinsmith and Kaplan, 1963). The opposite pattern was observed for low-arousal paired associates; those were best recalled immediately after encoding. Although, the effect of arousal on memory became stronger with time, it was influenced by the type of material and presentation rate (Kaplan and Kaplan, 1969, 1968). More recently, Sharot and Phelps (2004) presented participants with neutral and negatively-valenced arousing words at the periphery of a central (neutral) word and found that the EEM was larger when forced-choice recognition was tested after 24 hours than after 3 minutes (see Figure 1). Moreover, increased memory confidence and recollection (as measured by 'remember' judgments in a remember-know task; see Section 4.3) for negative versus neutral pictures were greater after 24 hours than after a few minutes (Sharot et al., 2007). These studies are particularly striking because they suggest that memory for emotional experiences can actually increase with time, whereas neutral memories are forgotten. It is more typical to find that the EEM is more robust on delayed tests, and is reduced, or entirely eliminated, on immediate tests (LaBar and Phelps, 1998; Schwarze et al., 2012; for a review, see Yonelinas and Ritchey, 2015).

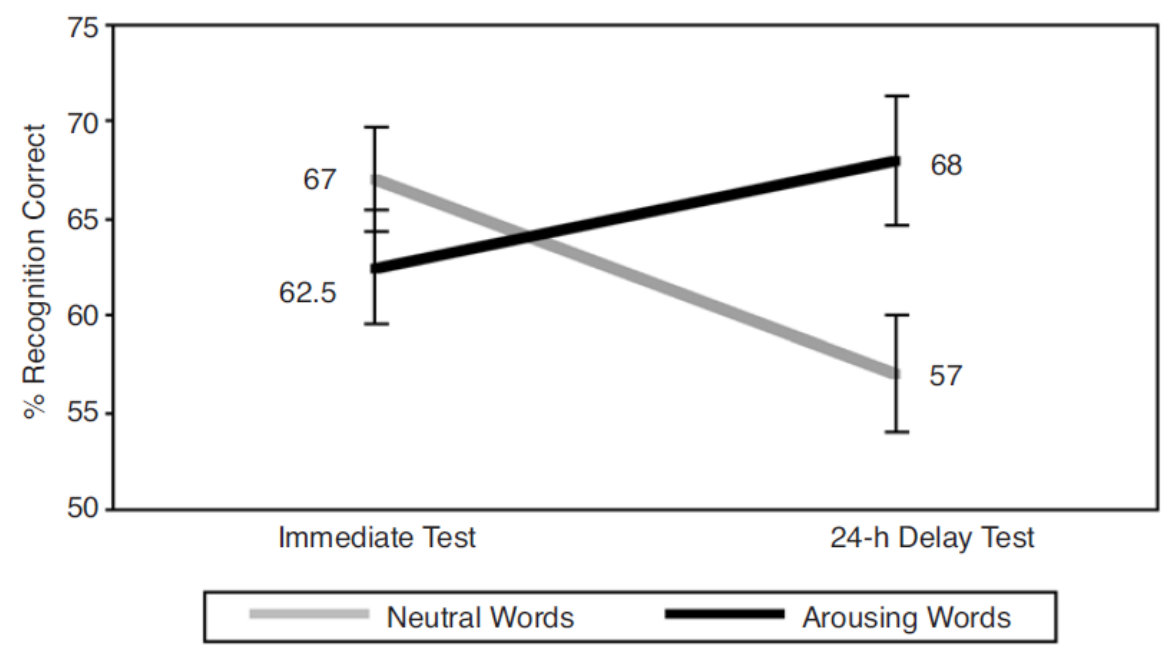

Figure 1. Recognition for neutral and negative words, presented at the periphery of a central (neutral) word, when tested immediately or 24-hours after encoding. Reprinted from Sharot, T., Phelps, E.A., 2004. How arousal modulates memory: Disentangling the effects of attention and retention. Cogn. Affect. Behav. Neurosci. 4, 294-306, with permission. 
Most researchers agree that the EEM is larger in delayed tests. This conclusion has crucial theoretical and applied importance, however, caution is warranted before it is accepted. We should be mindful that only a limited number of modern studies have tested EEM at two or more intervals. Those studies have employed a variety of methodologies and obtained a variety of patterns, and typically used only the 'vertical' method to interpret forgetting (Wixted, 1990). Clearly, meta-analytic evidence would be useful here. One meta-analysis published by Park (2005) sought to address this question, but suffered from several weaknesses. It used a problematic inclusion criterion, where 'arousal' was manipulated in highly variable ways (e.g., arousing stimuli, caffeine ingestion, white noise, learning in the morning vs. evening), did not break memory performance down by method (recall, hit rates), and observed - but did not address - considerable heterogeneity across the target articles. The authors made an attempt to account for the file-drawer problem where it may be difficult to publish studies that disagree with the dominant perspective that EEM is greater with time, by using a formula developed by Rosenthal (1991), but they did not contact researchers to request unpublished data. Preregistering studies on EEM with two or more test intervals would be particularly useful in solving the filedrawer problem. It will be important to relate conclusions about the time-dependent effects of arousal to previous work on factors that modulate human forgetting (Wixted, 1990) and to available evidence for dissociations between early and late episodic memory in humans (Dudai, 2004; Wixted, 2004).

\subsection{Post-Encoding Arousal Induction Alters Delayed Memory}

In humans, like in other animals, post-encoding interventions that increase adrenal hormone release can enhance delayed memory (LaBar and Cabeza, 2006; van Stegeren, 2008). Because such paradigms induce arousal after encoding is completed, they provide direct evidence that storage is key to attenuated forgetting of emotional memories, supporting the modulatedconsolidation model. For instance, after learning a list of neutral words, participants were exposed to an emotionally-arousing negative (dental surgery) or positive (comedy) video (Nielson and Powless, 2007). Performance on an old-new recognition test one week later was enhanced when either of the emotional videos was presented $0 \mathrm{~min}, 10 \mathrm{~min}$, or 30 minutes after 
the word list, but not when presented 45 min after the word list, demonstrating the timedependent effects of post-training arousal on consolidation.

Mather and Sutherland (2011) proposed that arousal during storage particularly influences stimuli that were prioritized at encoding. An efficient way of inducing arousal in humans is the cold-pressor test, a task in which participants must keep their arms submerged under ice water, and it is known to cause release of adrenal hormones (Cahill et al., 2003). Performing cold pressor stress after viewing a series of pictures improved incidental free recall, tested after one week, for emotional but not neutral pictures (Cahill et al., 2003; see Figure 2). Additional evidence was reported by Cahill and Alkire (2003) in which intravenous infusions of epinephrine produced dose-dependent improvements in an incidental picture free recall task performed one week after encoding: The largest dose of epinephrine produced the highest rates of recall. These memory effects were only observed for the first three slides (compared to the last three slides), demonstrating once again that the post-training levels of epinephrine interact with degree of arousal during initial encoding to modulate consolidation (Cahill and Alkire, 2003). Furthermore, when an adrenoreceptor antagonist (e.g., propranolol) was administered, a treatment that blocks hormonally-mediated amygdala activation (van Stegeren et al., 2005), these enhancing effects were eliminated (for a meta-analysis, see Lonergan et al., 2013). For example, in a classic study investigating the effects of propranolol on memory (Cahill et al., 1994), participants were injected with propranolol, and instructed to memorize a picture slideshow of either a neutral or emotional story, before the drug began exerting its effects. The adrenoreceptor antagonist entirely abolished the memory advantage for the emotional story in a delayed memory test, whereas memory for the neutral story remained unaffected. Individual differences are also found, where post-encoding stress enhances memory in those who were emotionally aroused at encoding (Abercrombie et al., 2006). Yet stress induction after encoding does not always selectively enhance delayed memory for emotional material (McCullough and Yonelinas, 2013; Trammell and Clore, 2014; Yonelinas et al., 2011). For example, post-encoding arousal, induced by skydiving, increased delayed recognition memory for neutral but not negative pictures in men, and had no influence on female participants' memory, relative to a non-stress control group. Free recall memory was not modulated in any way by the stress manipulation. 


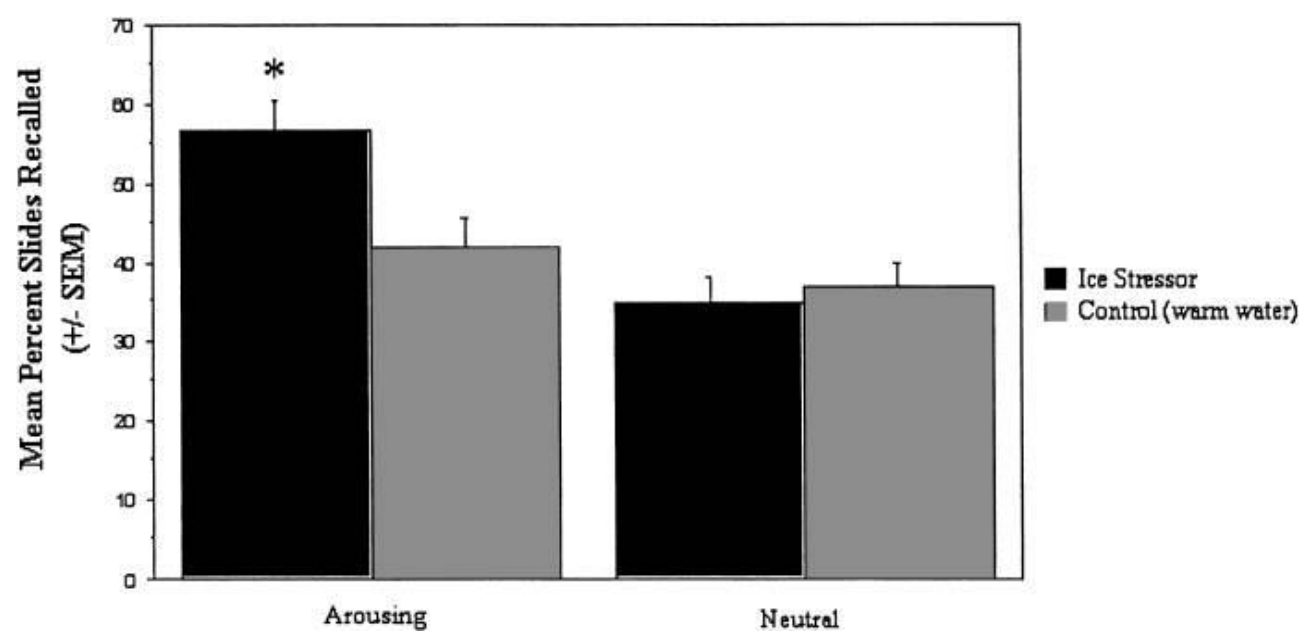

Figure 2. Free recall of negative and neutral pictures, tested after one week, in cold pressor stress and control groups $(* \mathrm{p}<.02)$. Reprinted from Cahill, L., Gorski, L., Le, K., 2003. Enhanced human memory consolidation with post-learning stress: interaction with the degree of arousal at encoding. Learn. Mem. 10, 270-274.

Together, these studies demonstrate that the release of stress hormones is a key contributor to enhanced delayed memory in humans, and that there is an interesting interplay between their levels at encoding and during storage. There are, clearly, some discrepancies in the literature between studies that do and do not observe stress-dependent EEM, and in the selectivity of the memory consequences of post-encoding arousal. To resolve these, it is important to consider the overlap between neurobiological circuits activated by the original experience and the arousal induction. Stress hormones are thought to enhance delayed memory only when they are released close in time and within the same context as the originally arousing experience, or when they activate the same circuits (Joëls et al., 2006). This hypothesis can explain why the smell of a predator increased spatial memory in mice, an effect that depended on the hippocampus and amygdala (Galliot et al., 2010), but exposure to a cat at a different context 30 minutes after encoding did not enhance spatial memory in rats (Park et al., 2008). This literature suggests that subtle differences in the experimental set-up in human studies - and even the way different participant groups experience the set-up - could have important differences on the consequences of post-encoding interventions.

\subsection{Central Role of the Amygdala in Human EEM}


Studies in animal models suggest that various effects of systemic and central arousal associated with emotional experiences culminate in amygdala activation. There is evidence that the amygdala also contributes to the EEM in humans. Lesion studies have shown that the EEM is greatly reduced or even completely eliminated when both amygdalae are damaged. Early work (Adolphs et al., 1997; Cahill et al., 1995; Markowitsch et al., 1994) found that delayed EEM (tested after 24 hours or more) was completely eliminated in three Urbach-Wiethe patients (SM046, B.P., C.P.) with selective bilateral amygdala damage (thought to center on the basolateral amygdala, in at least some cases; Klumpers et al., 2015). Follow-up work (Siebert et al., 2003) with ten Urbach-Wiethe patients found similar impairments in recognition memory for both positive and negative but not neutral pictures when tested after one hour. Although some patients with more widespread temporal lobe damage (affecting the amygdala, hippocampus, and adjacent areas) showed enhanced memory for taboo words over neutral words on immediate and one-hour delayed tests, there were no differential forgetting rates between the emotional and neutral stimuli (LaBar and Phelps, 1998; Phelps et al., 1998). This suggests that contrary to control participants that exhibit increased memory for taboo words over time, patients with amygdala damage show impaired consolidation of arousing events, but their encoding and retrieval processes must remain somewhat preserved in order to support immediate EEM (see Section 3). The important role of the amygdala in EEM can be contrasted with that of the medial temporal lobe (MTL): Lesions of the MTL reduce memory overall, but not the EEM effect when the amygdala remains preserved (Hamann et al., 1997; Hamann et al., 1997; Sharot et al., 2007). The amygdala and hippocampus therefore work independently although they interact when processing emotional information (Phelps, 2004).

There is also cumulative evidence from neuroimaging studies that amygdala activation during encoding modulates memory for emotionally-arousing stimuli. The subsequent-memoryparadigm consists of recording neural activity during the encoding of stimuli and calculating the 'difference due-to-memory' (Dm) effect, by subtracting brain activity associated with items that were later forgotten from those that were correctly remembered. This paradigm helps unravel whether amygdala activity is merely associated with the processing of emotional content, or reflects a genuine memory function. In an emotional subsequent-memory paradigm, subsequently-forgotten and -remembered emotional items should have the same brain processing of emotional features at encoding. Consequently, if a difference in brain activity associated with 
each category (forgotten vs. remembered) is reported, its origin is explained by differences in encoding processes. Dolcos and coworkers (Dolcos et al., 2004a) found that the amygdala was more strongly activated for emotional (including both positive and negative items) than for neutral pictures suggesting that its activity is associated with emotional processing (for consistent results across the literature, see meta-analysis from Murty et al., 2010). However, both the amygdala and the hippocampus were more activated by subsequently-remembered than by subsequently-forgotten emotional pictures on a cued-recall test performed 45 minutes after encoding. Moreover, the correlation between amygdala and hippocampal activity at encoding was greater for emotional than for neutral items. Taken as a whole, these results suggest that successful encoding of emotional items is predicted by the co-activation of the amygdala and the hippocampus (Dolcos et al., 2004a). Similar findings have been reported in favor of the coactivation of the amygdala and prefrontal cortex (PFC) sustaining emotional memory encoding (Dolcos et al., 2004b; Sergerie et al., 2005). Functional connectivity analysis revealed a greater connectivity between the amygdala (specifically the voxel maximally associated with enhanced memory for emotional videos) and lateral PFC during the encoding of emotional relative to neutral videos (Kilpatrick, 2003). Furthermore, connectivity between the amygdala and parahippocampal regions at encoding was more predictive of memory for negative pictures when tested after a 1-week delay than after a 20-min delay (Ritchey et al., 2008), an effect not observed with neutral pictures. This further reinforces the notion that emotional memories consolidate over time and that the amygdala is central to producing these effects. One point we should make here is that until recently, it has been difficult to examine amygdala activity with the spatial resolution needed to determine whether different sub-regions (e.g., the basolateral amygdala; BLA) are differentially involved in EEM, but this will undoubtedly change soon (for a template, see Leal et al., 2017).

Although many of these findings are highly suggestive of selective effects of the amygdala on storage, it is important to bear in mind that some of the effects of the amygdala on delayed EEM may well be observed in immediate memory tests. For example, Kensinger and Corkin (2004) tested memory after 10 minutes, and Dolcos et al. (2004a) after 45 minutes. Such effects may be attributed to amygdala-dependent changes in processing emotional stimuli at encoding, an issue we return to in Section 3. 


\subsection{The Modulated-Consolidation Model}

The modulated-consolidation model provides insight into how endogenous physiological processes associated with emotional arousal lead to changes in the brain that enhance memory for emotionally-arousing experiences over time. The model has two main tenets. First, changes in amygdala activity at the occurrence of an emotion, or within a short time-window thereafter, are necessary and sufficient to explain emotional influences on consolidation. Second, the amygdala does not itself store episodic memories, but influences memory for emotional material through its interaction with other brain areas, in particular the hippocampus. These claims are supported by an extensive literature, which has examined mechanisms sustaining consolidation of emotional material by stimulating or blocking neuronal activity occurring during an emotional experience (for reviews, see McGaugh, 2004, 2000; Paré, 2003; Roozendaal et al., 2008). The following sections will describe a few key pieces of evidence from animal studies in support of the modulated-consolidation model.

In the one-trial avoidance learning task an animal is placed in an aversive location, such as a brightly-lit arena or a high platform. When it escapes, for example to a lower or darker compartment, it is punished, typically with an electric shock. At test the animal is placed at the aversive location, and memory for the emotional experience is operationalized as the duration of time the animal stays there before escaping to the previously punished area. Animals that remember the punishment will take longer to escape the aversive location than those that have forgotten about the previous experience. In the one-trial version of the task, the animal is only exposed to the associated punishment once, after which memory for the event is tested later. Many parameters of this paradigm can be changed (e.g., the number of learning trials, the duration and number of test trials, and the foot shock intensity), rendering it adaptable to different research questions. In addition, one-trial avoidance (Mitsushima et al., 2011), as well as Morris water maze learning (D’Hooge and De Deyn, 2001), are related to contextual conditioning and depend on the hippocampus, reflecting a similar medial temporal lobe network as the one attributed to episodic memory in humans.

Direct electrical stimulation to the amygdala immediately after training impaired one-trial avoidance learning after intense foot shock (Gold et al., 1975a), but improved learning after mild foot shock (Gold et al., 1975b) when tested after 24 hours, demonstrating that the modulatory 
effects of the amygdala on memory consolidation depend in part on the degree of arousal (i.e., shock intensity) during encoding. Amygdala stimulation has also been investigated using the Novel Object Recognition (NOR) task, which, rather than motivate rodents through reinforcement, relies on their tendency to explore unknown objects more than familiar ones. Based on this principle, if an animal is presented with two objects, one that is new and one that was previously shown, the animal should spend more time exploring the novel object if it recognizes the familiar one. A brief stimulation of the amygdala immediately after the animal has viewed an object enhanced delayed, but not immediate, memory for specific objects explored in the NOR test (Bass et al., 2012).

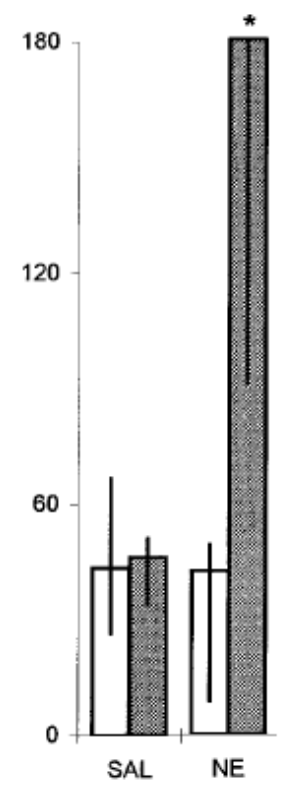

Figure 3. Effects of different drugs on mean escape latency in a one-trial inhibitory avoidance task in rats. Intra-amygdala injections of norepinephrine enhanced learning when tested 24 hours (gray bars), but not 1.5 hours (white bars) after training, compared to a control (saline) group. $\mathrm{NE}=$ norepinephrine; SAL = saline $(* \mathrm{p}<.02)$. Modified from Bianchin, M., Mello-e-Souza, T., Medina, J.H., Izquierdo, I., 1999. The amygdala is involved in the modulation of long-term memory but not in working or short-term memory. Neurobiol. Learn. Mem. 71, 127-131, with permission.

Adrenal stress hormones (i.e., epinephrine and cortisol) are released during emotionallyarousing events. Systemic injections can mimic these effects. For example, post-training administration of epinephrine improved NOR retention (as measured by exploration preference) 
when tested after 1.5-, 24-, and 96-hour delays (Dornelles et al., 2007). Inter-individual differences in rats were also observed, whereby the rats that released the greatest endogenous epinephrine after training performed the best on a one-trial inhibitory avoidance task 24 hours later (McIntyre et al., 2002). The BLA is crucial for the memory-enhancing effects of both epinephrine and corticosterone (McGaugh, 2000; McGaugh and Roozendaal, 2002; Roozendaal, 2002 , 2000), although these two hormones involve different pathways into the amygdala (Roozendaal and McGaugh, 2011). Adrenergic activation is thought to underlie the emotional arousal that is necessary for producing glucocorticoid enhancement of memory (Roozendaal et al., 2006). For example, when emotional arousal was reduced during learning (as is the case after extensive habituation; De Boer et al., 1990), corticosterone administration no longer enhanced memory of the objects 24 hours later (Okuda et al., 2004). The adrenergic effects on memory are time-dependent: Epinephrine selectively enhanced performance on the one-trial avoidance learning task when tested after 24 hours, but not when tested immediately after or 1.5 hours after training (Bianchin et al., 1999; see Figure 3).

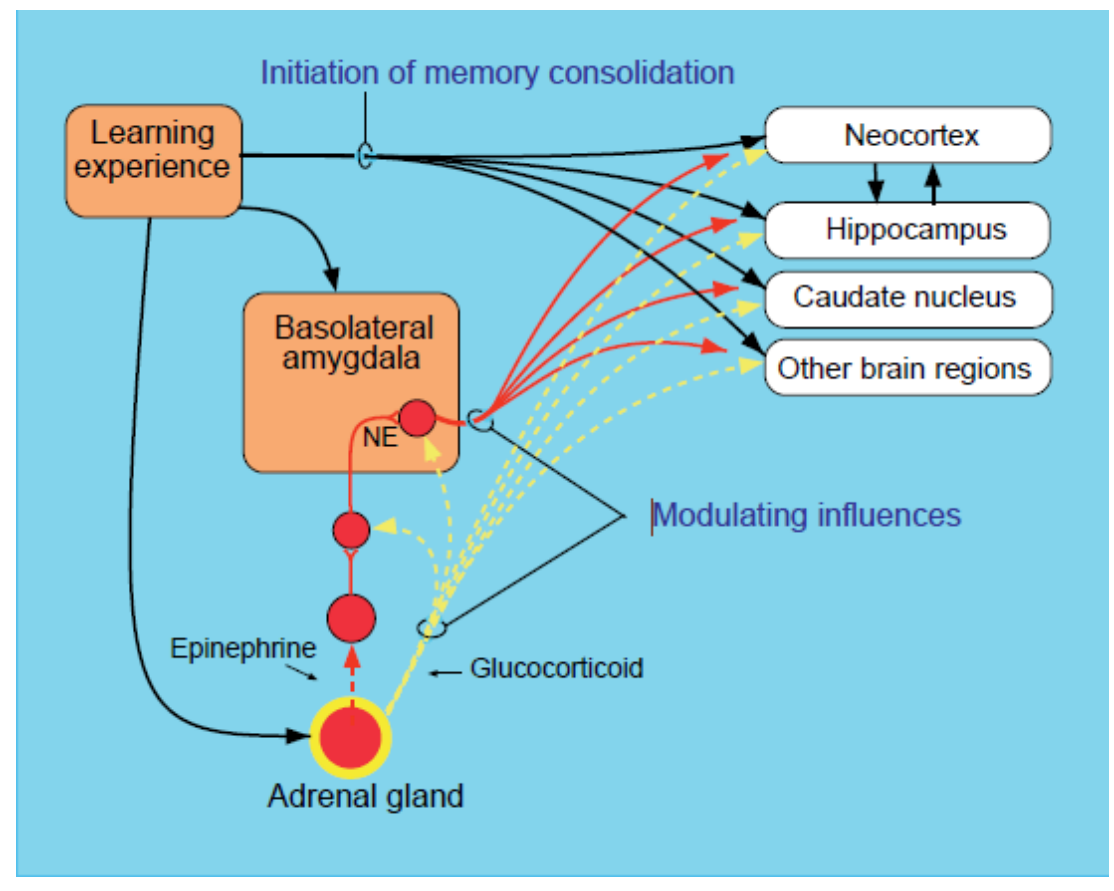

Figure 4. Hypothetical memory-modulatory mechanism for emotionally arousing events. When an event has an emotional charge, the amygdala is activated directly and also indirectly, via stress hormones. Amygdala activation modulates the activation of highly-connected memory systems. NE = norepinephrine. Reprinted from McGaugh, J.L., 2000. Memory--a Century of Consolidation. Science 287, 248-251, with permission. 
But how can the amygdala aid in the consolidation of emotional memories when it is generally not considered a site for declarative memory storage? There is substantial evidence that the amygdala modulates memory traces stored in other brain regions, particularly through the BLA's projections to the hippocampus (McGaugh, 2000; see Figure 4). An influential theory, called 'synaptic tagging and capture' (Frey and Morris, 1998), describes the amygdala as being able to 'tag' or 'mark' an arousing event as important to remember by strengthening the synaptic plasticity in the hippocampus (Richter-Levin and Akirav, 2003). More specifically, this molecular tag would allow the activated synapses to capture more newly synthesized proteins to ensure that early long-term potentiation (LTP) prolongs into late LTP, leading to long-lasting plasticity in the activated synapses. Adrenergic and cholinergic afferents into the hippocampus could be responsible for activating the molecular cascade regulating protein synthesis, ensuring that the plasticity-related proteins are specifically inserted into the emotionally-tagged synapses. The modulatory influences of the amygdala occur within a specific time window after encoding. When the BLA of rats is stimulated within 30 minutes (before or after) tetanization of the dentate gyrus, protein synthesis is reinforced and early LTP persists into late LTP (Frey et al., 2001). Similarly, intra-amygdala injections of amphetamine enhanced 24-hour water maze learning in rats when administered immediately after training but not 2-hours later (Packard and Teather, 1998; see Figure 5A). Crucially, while inactivating the hippocampus (with a lidocaine injection) before the delayed test impaired memory, inactivating the amygdala with the same procedure had no effect on memory (see Figure 5B), supporting the suggestion that it plays a time-limited role in memory modulation rather than involved in memory storage. Similarly, discrimination avoidance learning was impaired when the amygdala was inactivated (by muscimol) 15 minutes prior to or immediately after training, but not when it was inactivated 15 minutes before the test the next day (Ribeiro et al., 2011). These findings suggest that the amygdala is able to modulate early LTP mechanisms, tagging the synapses that should later be reinforced, but is not itself responsible for storing memories and ensuring the prolongation of early LTP into late LTP. 
A
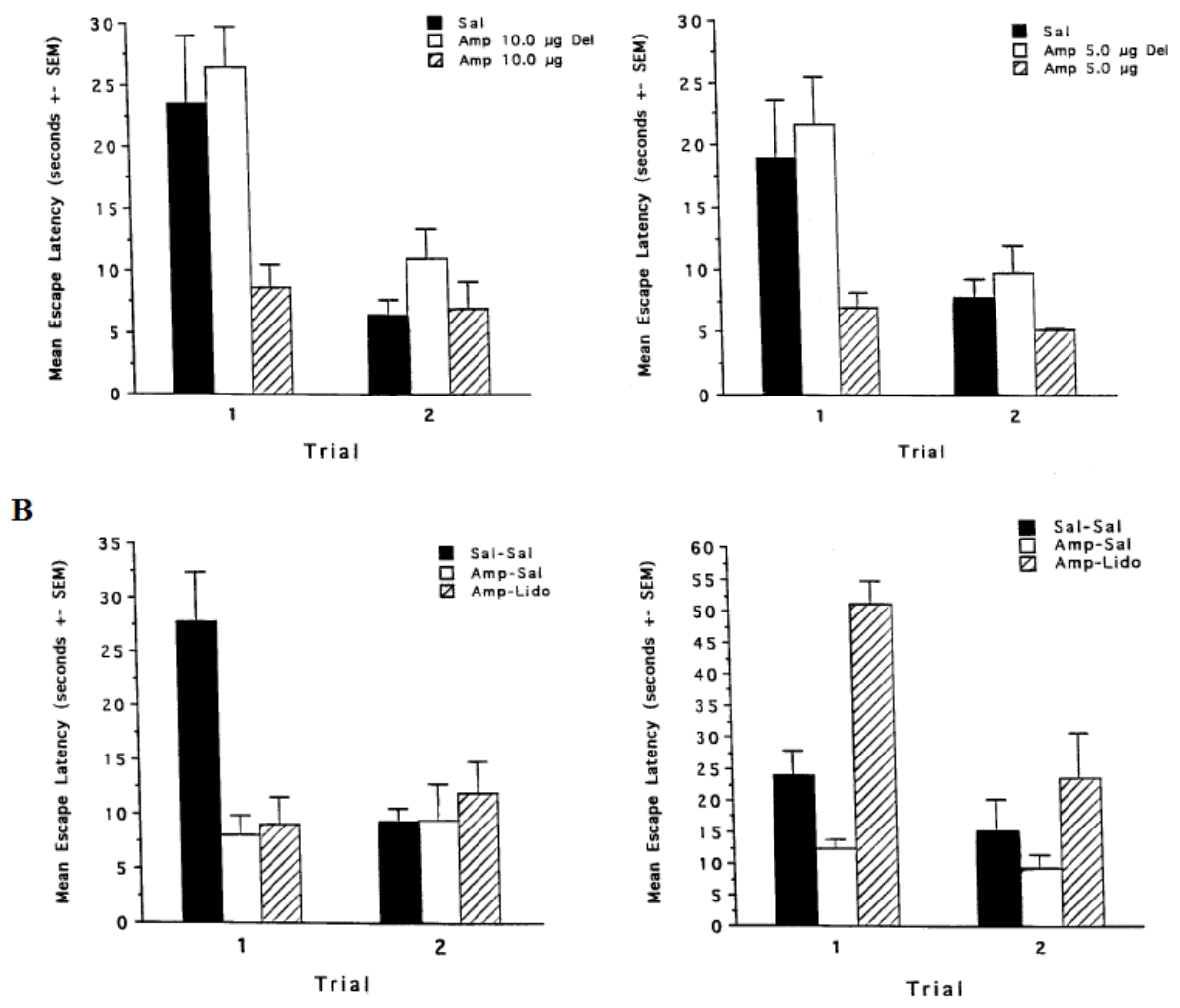

Figure 5. Effects of post-training amphetamine (A) and preretention (i.e., prior to a 24-hour delayed test) lidocaine (B) injected into the amygdala (left panels) or hippocampus (right panels) on two retention trials 24 hours after training. Intra-amygdala and intrahippocampal injections of amphetamine (A) enhanced water-maze learning in rats when administered immediately after (diagonal bars) but not 2-hours after (white bars) training, compared to a control saline group (black bars). Inactivating the amygdala (with lidocaine preretention) did not reduce the enhancing effects of post-training amphetamine on learning (B, left panel), whereas inactivating the hippocampus (with lidocaine preretention) impaired learning (B, right panel). Modified from Packard, M.G., Teather, L., A., 1998. Amygdala modulation of multiple memory systems: Hippocampus and caudate-putamen. Neurobiol. Learn. Mem. 69, 163-203, with permission.

The modulated-consolidation model provides a reasonable account of the hormonal and neural processes that produce increased memory for emotionally-arousing events in animals and humans alike. The key role of the amygdala, and the similarities between the animal and human findings in studies of post-encoding arousal induction, further emphasizes the evolutionary advantage of prioritizing the retention of emotional experiences (see Chapters 7 and 28). Indeed, the modulated-consolidation model became the foundational interpretation of contemporary 
studies on the EEM effect in humans. Although the model is consistent with the evidence considered so far, it does not provide a comprehensive account of the complex nature of emotional memory in humans.

Several key distinctions between animal and human research must be considered, namely the operationalization of episodic memory and the selectivity of what arousal enhances. The paradigms that are employed to test emotional memory in animals and humans differ enormously. Animal studies often use 'primary' appetitive or aversive stimuli - for example, delivery of fruit juice or foot shock, and require the organism to learn an association between that stimulus and some aspect of context. The majority of animal studies examine memory for a single situation, as in the one-trial avoidance learning task, rather than comparing an emotional and a neutral situation. Moreover, the task is often the only experience the animal has in life and is therefore inherently of high priority. By contrast, human studies usually use long lists of symbols of primary and secondary appetitive (e.g., images of food or money) and aversive stimuli (e.g., images of snakes or guns) to simulate a psychophysiological response similar to that in real-life events; any effect of emotion in such paradigms is necessarily focal in time and space, as discussed in Section 4. Performance outcomes in humans are each participant's ability to probe memory for the symbol itself (Do you recognize this image of food?) with little dependence on contextual learning; the nature of the test matters very much to EEM in humans, with no equivalent in animals. Paradigmatic animal and human studies thus differ on four important respects: the personal relevance of the emotional experience; its distinctiveness within the global and the local context of the participant; the nature of the memorandum (item vs. itemcontext links); and the degree to which we can be sure that a test probes episodic memory specifically. When animal and human studies match better, they sometimes yield surprising effects (Bass et al., 2014; Schwarze et al., 2012).

Recently, an alternative model for the human findings has been proposed. According to the emotional binding account of memory (Yonelinas and Ritchey, 2015) emotional memories persist more than neutral memories because the trace representing the emotional content of an episode is stored permanently in the amygdala, and retained there better than hippocampal traces, which represent other contextual aspects of the episode. The suggestion is that amygdala storage is more stable than hippocampal storage due to increased neurogenesis and cell death in the 
hippocampus, or a greater "active decay process" (Yonelinas and Ritchey, 2015, p. 263). The emotional binding account therefore predicts that amygdala lesions would always be detrimental to the EEM, even if they occur just before retrieval, after consolidation has occurred. This prediction contrasts with the prediction of the modulated-consolidation model, which proposes that the amygdala plays a time-limited role in modulating the consolidation of hippocampal memory traces, and is no longer needed once consolidation there is completed. Here the gap between the animal and the human paradigms becomes important. Although existing evidence supports the predictions of the modulated-consolidation model (Packard and Teather, 1998; Ribeiro et al., 2011), it would be interesting to check that they hold in animal tasks that are more similar to those carried out in humans. Decisive evidence from humans requires that we test memory in participants with amygdala damage which has occurred hours, days, or longer after the target emotional (and control neutral) experiences were encoded, something that is impossible to achieve in laboratory studies. Recently, one study found that Alzheimer's patients recall fewer emotional autobiographical events, an effect that was correlated with amygdala (and hippocampal) volume - in agreement with the emotional binding account (Philippi et al., 2015).

\subsection{Effects of Sleep on Delayed EEM}

One aspect of a long retention interval is that participants sleep between study and test, which can influence both emotion processing and emotional memory. Cumulative evidence has revealed various interactions between sleep and affective states and neural responses to emotional stimuli. First, affective disorders are accompanied by profound changes in sleep architecture. For example, REM-sleep latency is reduced in depressed patients (Armitage, 2007; Gottesmann and Gottesman, 2007; Tsuno et al., 2005), whereas it is increased by stress (Cheeta et al., 1997). Second, experimental modulations of sleep alter affective states. In healthy adults, sleep deprivation has a major negative impact on mood (see Pilcher and Huffcutt, 1996). By contrast, sleep deprivation protocols in depressed patients improve mood state the next day (Giedke and Schwärzler, 2002). Third, spontaneous activity in neural networks thought to be linked with emotion, including the amygdala, is observed during REM-sleep (Maquet et al., 1996). These findings corroborate behavioral evidence showing that emotional reactions to unpleasant pictures increased after a period of REM-sleep (Wagner et al., 2002a). Fourth, sleep changes the neural response to emotional information. For example, amygdala activity in 
response to viewing emotionally-arousing pictures (but not neutral pictures) was increased when participants watched them after a night of sleep deprivation compared to a night of sleep (Yoo et al., 2007), resulting perhaps from the inability to inhibit amygdala activity because of lost functional connectivity between the amygdala and medial prefrontal cortex following sleep deprivation (Walker and van der Helm, 2009; Figure 6). While sleep deprivation has myriad implications, REM-sleep may be particularly important to these changes in neural responses. In another study, amygdala activity was once again reduced when viewing unpleasant pictures for a second time when preceded by a period of sleep compared to a period of wakefulness, a reduction that was significantly correlated with gamma activity during REM-sleep (van der Helm et al., 2011). Gamma changes during REM-sleep have been identified as an index of adrenergic activity reduction (Cape and Jones, 1998), suggesting the reduced maintenance of emotional information during sleep.
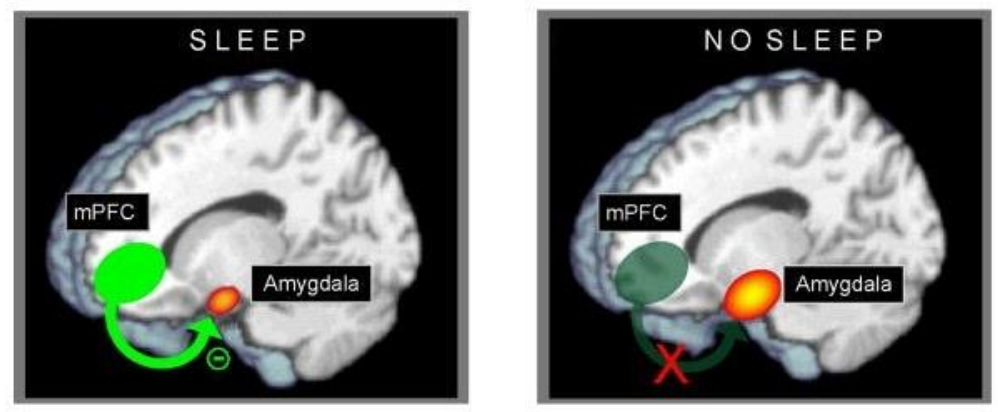

Figures 6. Impact of sleep deprivation on the connectivity between the amygdala and the medial prefrontal cortex (mPFC). Modified from Walker, M.P., van der Helm, E., 2009. Overnight therapy? The role of sleep in emotional brain processing. Psychol. Bull. 135, 731-748.

Converging literature has directly assessed the effects of sleep on emotional memory consolidation. These studies suggest that sleep selectively improves long-lasting declarative memory of emotionally-arousing events (Wagner et al., 2001; Wagner and Born, 2008), even when sleep duration is short (e.g., in nap studies; Pace-Schott et al., 2011a; Wagner et al., 2006). Effects of sleep on emotional memory are stable over long periods. Reduced memory for emotional information following sleep deprivation has been found for encoding-test delays of only a few minutes to 72 hours (Sterpenich et al., 2007), one week (Atienza and Cantero, 2008), and even 4 years (Wagner et al., 2006). In the latter study, participants had studied neutral and emotional texts, after which half of the participants slept immediately and the other half was kept 
awake for 3 hours. After 4 years, a recognition task demonstrated that memory for the emotional - but not neutral - text was better for the sleep group compared to the sleep-deprivation group. Both recollection and familiarity for emotional material also improves with sleep, compared to sleep deprivation (Hu et al., 2006). Successful recollection of emotional pictures compared to neutral pictures (in the remember-know task) was also associated with greater activation of the amygdala, hippocampus, and ventromedial prefrontal cortex in participants that slept versus those that were deprived of sleep following learning (Sterpenich et al., 2007). This change in the neural processing of emotional recollection remained 6 months later (Sterpenich et al., 2009, 2007). In addition, Payne and Kensinger (2011) found that the sleep condition was associated with a partially different neural network than the one activated in the sleep-deprived group.

Importantly, memory consolidation that occurs during sleep depends on specific sleep stages (for reviews, see Diekelmann and Born, 2010; Rauchs et al., 2005). Whereas Slow-Wave Sleep (SWS) benefits episodic memory in general, REM-sleep specifically benefits EEM. Wagner et al. (2001) found that the recall of emotional, but not neutral, stories was improved by REM-sleep. It is interesting to note that sleep plays a similar role as emotional arousal on memory: it selectively preserves emotional gist information and deteriorates non-emotional peripheral details (Payne et al., 2008; see Section 4.1). Nishida and coworkers (Nishida et al., 2009) showed that the EEM was correlated with the length of REM-sleep and the magnitude of prefrontal theta activity during this particular sleep stage. Furthermore, Payne and coworkers recently found that the level of endogenous cortisol at encoding interacts with sleep to enhance emotional memory consolidation (Bennion et al., 2015). Participants with the highest cortisol levels at encoding produced the largest EEM effects, but only after a night of sleep; a wake group that did not sleep during the day showed no relationship between cortisol levels and subsequent memory. This finding suggests that elevated cortisol may help 'tag' encoded information as important to remember throughout the day, enabling sleep-based consolidation to solidify that information. This hypothesis has to be more extensively assessed but so far the findings are congruent with the modulated-consolidation model of EEM. Together, these results demonstrate that sleep, especially REM-sleep, may offer a neurobiological state that is especially well-suited to the preferential storage of emotional memories. 


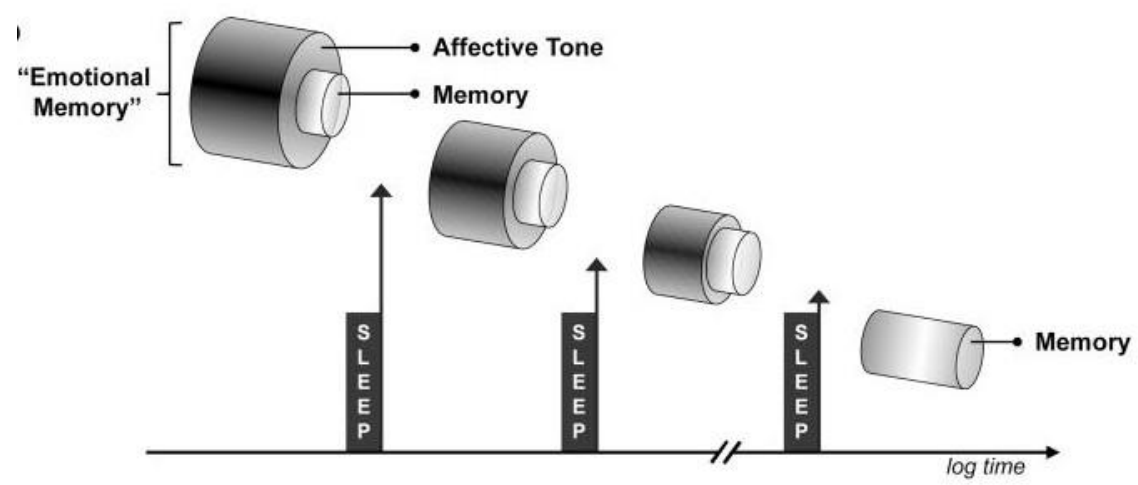

Figure 7. Through multiple iterations of this REM mechanism across the night and/or across multiple nights, the long-term consequences of sleep-dependent reprocessing improves the retention of salient information that was tagged as emotional at the time of learning. Modified from Walker, M.P., van der Helm, E., 2009. Overnight therapy? The role of sleep in emotional brain processing. Psychol. Bull. 135, 731-748.

Based on this consistent body of evidence, Walker and Van der Helm (2009) developed the 'Sleep to Forget Sleep to Remember' (SFSR) model. According to SFSR, several successive REM-sleep periods are required to support the dis-association of the content and the affective value of a memory trace (note that this idea is somewhat contradictory to the notion that the affective value and the content are bound indefinitely, as in the emotional binding account of memory in the previous section). The authors argue that two processes are initiated during REMsleep: one dedicated to remembering the emotional event and the other to reducing its emotional tone (Figure 7), and that physiological and neurochemical conditions during REM-sleep are optimal to allow these two processes to occur. Until recently most research focused on REMsleep, but now growing evidence shows that SWS, as well as Stage 2 sleep and its cerebral correlates are involved in non-emotional memory consolidation, and may play a role in emotional memory too (Deliens et al., 2013; Pace-Schott et al., 2013, 2011b; Wagner et al., 2002b). The idea of a dual process associated with different sleep stages should therefore be explored in more depth.

\section{Cognitive Factors Mediating Early EEM}

Section 2 explained how emotional arousal benefits late LTM. Yet it is probably adaptive to remember events that are important to survival as soon as they occur. If you see your friend a 
couple of minutes after their dog has bitten you, it would be helpful if your memory for the bite was good, so that you could avoid the dog this time. As Cahill and McGaugh (1998) acknowledge in their classic paper, the advantage of emotional stimuli in tests of early LTM requires a different explanation from the explanation the modulated-consolidation model offers for delayed EEM.

Emotional experiences differ from neutral ones in many respects. Many of the features associated with emotional experiences - from appraisals and physiological responses to feelings - are both unexpected and unique, namely, not shared by neutral experiences. On the one hand, these unique features help emotional experiences stand out relative to the context in which they occur, and relative to other, neutral experiences stored in long-term memory. On the other hand, they also help people notice links between different emotional experiences. Substantial evidence suggests that emotional stimuli can capture additional attention resources bottom-up through unique mechanisms, unavailable even to the most important neutral stimuli (Pourtois et al., 2013). In most natural circumstances, these aspects of emotional experiences would give rise to alterations in the cognitive processes involved in their encoding and retrieval. Immediate EEM is likely mediated by such differential encoding processes (Christianson, 1992; Hamann, 2001; Mather et al., 2015; Mather and Sutherland, 2011; Schmidt and Saari, 2007; Sommer et al., 2008; Talmi, 2013). We discuss some of the key aspects of encoding emotional events (Sections 3.1-3.3), ending with a reflection on their joint contribution (Section 3.4). Of course, over time, arousal-modulated consolidation might further influence memory for emotional information.

While emotion can alter cognitive processes at encoding and retrieval, these cognitive processes themselves are not unique to emotional experiences. Clearly, however, the constellation of altered cognitive processing is part and parcel of the 'flux' of emotion (Lazarus, 1991), perhaps even inherent to the appraisal of a situation as emotional. The focus in this section on the mutual effects of emotion and cognition on each other does not imply that emotion and cognition are separate conceptually or neurobiologically (Barrett, 2014; Pessoa, 2013).

To understand fully how emotion changes encoding and retrieval we need to develop an understanding of the impact emotion has on aspects of cognition that are relevant to memory, and to demonstrate that those aspects of cognition do in fact mediate the EEM. One challenge here is that when the retention interval is long it is difficult to dissociate the influence of emotion 
on encoding and retrieval from its influence on consolidation. This challenge is exacerbated by the lack of clarity on the time frame of early and late long-term memory consolidation in humans (Dudai, 2004; Kandel et al., 2014; Wixted, 2004). To minimize modulated-consolidation effects it is simpler, therefore, to test memory fairly soon after encoding. Therefore, in this section we focus on memory tests that employed retention intervals of less than one hour, an arbitrary threshold, which is nevertheless consistent with the notion that the type of consolidation emotion is thought to influences in the modulated-consolidation model is slow consolidation into late LTM, a process thought to be dependent on de-novo protein synthesis, and to last several hours (Kandel et al., 2014).

\subsection{Emotional Stimuli Capture Attention}

The effect of emotion on attention has commanded much interest. Some emotional stimuli, such as snakes (Öhman et al., 2001; Öhman and Mineka, 2001) and fearful faces (Vuilleumier et al., 2003) may be attended preferentially because they are endowed with features thought to have hard-wired salience. Others, such as neutral stimuli conditioned with reward or aversion, acquire salience through learning (Phelps and LeDoux, 2005) or even through verbal instructions (Phelps et al., 2001). 


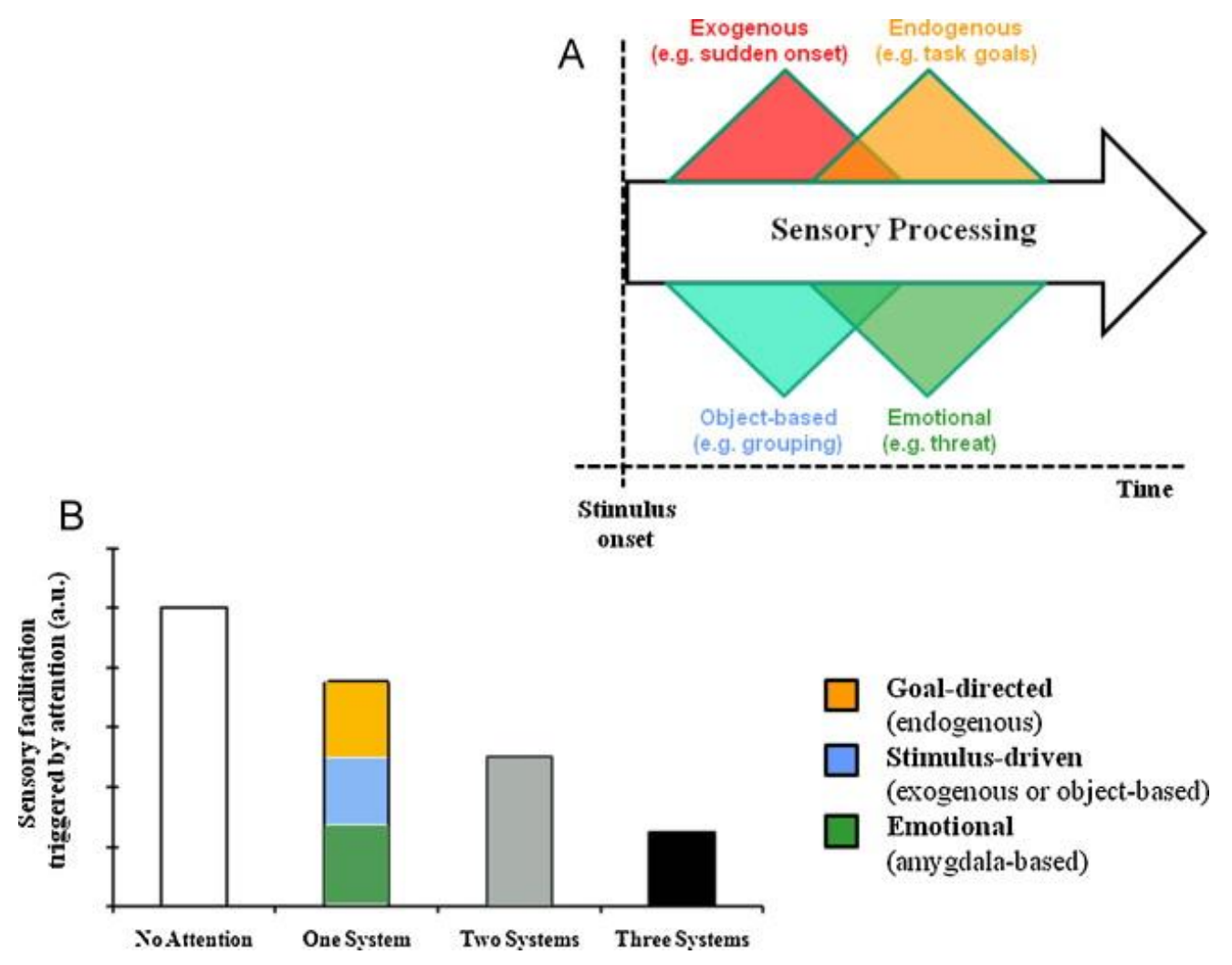

Figure 8. Exogenous, endogenous, object-based, and emotional systems are able to modulate perceptual processing through distinct neural circuits, but may influence the same processing network (e.g., striate and extrastriate visual cortex). Each system may be triggered by different sensory cues and may influence processing at different time frames although they may partly overlap (A). The schematic graph (B) illustrates the amount of sensory facilitation when one, two, or three systems guide attention control. An additive effect of the three systems appears when they are all driven toward processing the same stimulus; whereas interference occurs when they independently compete for resources. Reprinted from Pourtois, G., Schettino, A., Vuilleumier, P., 2013. Brain mechanisms for emotional influences on perception and attention: What is magic and what is not. Biol. Psychol. 92, 492-512, with permission.

Christianson (1992) has distinguished between pre-attentive processes, which lead to preferential selection of emotional stimuli and entail an orienting response and an interruption to other ongoing processing, and post-attentive processes, which enhance the perception of these stimuli. The evidence that has accumulated since then had generally supported these suggestions (for an excellent review, see Pourtois et al., 2013; Figure 8). For example, emotional words have a superior ability to escape the attentional blink (Anderson, 2005). Neuroimaging data show that neural responses to emotional stimuli is greater than the response to emotional stimuli and that this effect occurs very early, allowing them to gain access to higher cognitive processing stages. 
For example, stronger activation to fearful faces predicted better detection (Kouider et al., 2009; for review, including possible valence-related differences, see Pool et al., 2016; Yiend, 2010). Once activated, emotion-dependent attention can change the way that subsequently presented neutral stimuli are processed (Fox et al., 2002; Mogg and Bradley, 1999; Phelps et al., 2006). Once again, the amygdala plays a key role at encoding by tagging information that should be prioritized by both perceptive and attentional systems. The amygdala receives information about the emotional significance of a stimulus very early in stimulus processing and through feedback connections facilitates attention and later perception of emotional events (Davis and Whalen, 2001; Mather et al., 2015). Since perceptive and attentional systems have a limited capacity, peripheral (i.e. non emotional) information receives less perceptive and attentional resources, ensuring that important stimuli receive adequate processing resources (see Section 4.1). This distribution of resources is often defined as the 'weapon focus' effect (Loftus et al., 1987) indicating that a threating situation is associated with a high level of processing at the expense of non-affective information presented at the same time. When top-down (voluntary attention) is also involved, feedback connections between the amygdala and fusiform and visual cortex can produce additive effects and further enhance the processing of emotional stimuli. To illustrate, the amygdala enhances activation in the fusiform face area when exposed to fearful faces, yet fusiform activation will be even greater when the faces are task-relevant than task-irrelevant (Vuilleumier and Driver, 2007). The amygdala is thus able to produce a gain control effect in sensory-processing regions, akin to but separable from fronto-parietal systems involved in topdown and bottom-up attention to neutral stimuli (Corbetta and Shulman, 2002). Although the amygdala will certainly respond to arousing stimuli (as discussed within the framework of the modulated-consolidation model, see Section 2) the work on emotion and attention also aligns well with appraisal theories of emotion, where the amygdala is thought to be involved in appraising stimuli as goal-relevant (Sander et al., 2003). For example, in one study (N'Diaye et al., 2009) the amygdala was more strongly activated when fearful faces gazed away from the observer, and when angry faces gazed directly at the observer, the more threatening conditions. That these modulatory processes are obligatory is evident in the reliable accompanying costs to other tasks. For example, emotional words slow down font color naming, reading and lexical decisions (Algom et al., 2004), and emotionally-arousing words and pictures worsen 
performance on emotionally-neutral concurrent tasks even when participants are explicitly instructed to ignore them (Kensinger and Corkin, 2004; Kern et al., 2005; Talmi et al., 2007b).

Emotional stimuli may also induce more prolonged attentional effects, by inducing an arousal state. This is evident, for example, when participants' reading times of neutral words increase when emotional words are presented in the same block (Algom et al., 2004; McKenna and Sharma, 2004; Schmidt and Saari, 2007). Arousal-biased competition theory (Mather and Sutherland, 2011) suggests that arousal states increase the competitive advantage of stimuli (neutral or emotional) that are assigned priority. Such effects are thought to be dependent on altered noradrenergic function and interactions of noradrenaline with glutamate (Mather et al., 2015). For example, when faces were salient and places were not, hearing a threatening sound increased activation in the fusiform face area to the faces but decreased activation in the parahippocampal place area to places (Lee et al., 2014). This view suggests that when in an arousal state people orient towards certain external stimuli, in an effort to ensure that they detect the most important information. Arousal states are therefore different from other forms of cognitive load, where people attend to internal representations (Chun et al., 2011). While cognitive load makes it difficult to maintain focus on task-relevant stimuli, emotional arousal enhances performance on target stimuli at the expense of distracting tasks and stimuli, behaving more like perceptual load in load theory (Lavie, 2005).

These various changes in attention allocation, from selection and modulation to vigilance, are likely to have consequences for the way stimuli are remembered later on. Ultimately, the amount of attention dedicated to processing an event should affect subsequent memory for the event. Talmi and colleagues have used dual task paradigms to show that attention mediates the effect of positive emotion on memory (Talmi et al., 2007b), and that attention also mediates the effect of negative emotion on memory once other factors are controlled (Talmi and McGarry, 2012). For example, in one experiment participants were instructed to dedicate either $1 \%$ or $50 \%$ of their attention to the target pictures and the remainder on the secondary task (Talmi and McGarry, 2012). Here, attention was equal for negative and neutral images when encoded with $1 \%$ and $50 \%$, respectively, as indicated by similar accuracy and latency measures on the secondary task. The EEM in free recall was eliminated when comparing these two critical conditions in which attention was controlled. By contrast, the EEM appeared when comparing 
negative and neutral items that were given the same instructions to encode with either $1 \%$ or $50 \%$, where attention was preferentially allocated to emotional stimuli.

Despite being intricately related, studies of overt attention give rise to enough negative findings to give pause. In eye-tracking studies, length of gaze toward an object does not always predict subsequent memory. For instance, memory for negative images may increase despite longer fixations toward positive images (Gülçay and Cangöz, 2016; for similar effects after a one-week delay, see Humphreys et al., 2010). In addition, memory for central information in an emotional context was enhanced relative to a neutral context, despite shorter eye fixation latency and duration (Kim et al., 2013; see also Mickley Steinmetz and Kensinger, 2013). Moreover, mediation analysis revealed that the number of eye fixations toward pictures only partially accounts for the emotionally-enhanced memory for central information (Riggs et al., 2011). Taken together, differential attention to emotional stimuli explains part of the immediate EEM, although, as we will see in the following section, it does not provide a sufficient explanation for the enhancing effects of emotion on early LTM. Attending to emotional stimuli can also have implications for neighbors in space and time, which we discuss in Section 4.

\subsection{Emotional Stimuli are Semantically Inter-Related}

Memory improves when to-be-remembered stimuli are organized within a clear structure or schema, or when we organize stimuli ourselves by noticing the relationship between individual items (Alba and Hasher, 1983). Organization supports a more elaborative encoding of individual items and the processing associations between them (Einstein and Hunt, 1980; Hunt and Mcdaniel, 1993) and between them and the overall context (Kahana et al., 2008). During retrieval items that are related to each other are more accessible (Polyn et al., 2011; Steyvers and Griffiths, 2008).

The effects of organization are relevant to EEM because in the majority of research in this field the emotional stimuli are more inter-related than corresponding neutral stimuli (Talmi and Moscovitch, 2004). For example, participants rate images of a burning house, an injured woman and a starving child as more inter-related than neutral images of the same objects and people. One of the challenges for research on emotional memory is to quantify the semantic cohesiveness of stimuli so that the effect of organization on EEM can be studied. While several association norms exist for word stimuli, association norms for more emotionally-arousing 
stimuli such as taboo words and emotional pictures are currently unavailable. Another difficulty in studying the effect of organization on emotional memory is that while semantic relatedness can, in principle, be controlled by the experimenter, organization cannot be directly observed (Manning and Kahana, 2012). This is an issue for research on emotion because the evaluative dimension of stimuli is more easily discerned than other stimulus dimensions (Pratto and John, 1991), so it may be easier for participants to notice that emotional pictures were presented than to grasp the category membership of matched neutral stimuli. An additional issue is that emotional stimuli belong to several sub-categories. The dimension of emotional valence is relevant to organization effects because positive concepts (e.g., words) appears to be more closely related in memory than negative information (Unkelbach et al., 2008). This may result in part from the greater representation of negative over positive emotions in memory (Baumeister et al., 2001), which may lead to a larger and ultimately more diverse 'negative' cluster. Referred to as the density hypothesis (Koch et al., 2016; Unkelbach et al., 2008), this general valence asymmetry has been reliably found for a wide variety of stimuli and tasks and may require additional work to equate the organization of positive and negative stimuli.

In order for organization to influence the EEM there needs to be sufficient time to encode the meaning of stimuli and process relations between stimuli. Relational processing takes time; the greatest memory advantage for semantically related words occurs when the words are presented for $6 \mathrm{~s}$ than when presented for under 2s (Folarin, 1981). Organization effects may be less apparent when attention resources are limited (Talmi and McGarry, 2012), and the attention allocated to specific stimuli could modulate the effect of organization. For example, more arousing stimuli attract more attention (Kensinger and Corkin, 2004), which may explain why the EEM was not abolished in studies using taboo words and pictures even when organization was controlled (e.g., Buchanan et al., 2006; Houwer and Hermans, 1994; Kensinger and Schacter, 2006a; Talmi et al., 2007b). In addition, negative stimuli may more deeply encoded than positive stimuli, preferentially enhancing the associative links them (Baumeister et al., 2001). Of the studies comparing positive and negative stimuli, no difference in memory was observed when using highly-arousing words (Kensinger, 2008) or pictures (Talmi et al., 2007b), although increased memory for negative over positive stimuli did appear with less arousing stimuli (Kensinger, 2008) and higher task demands (Talmi et al., 2007b). Beyond time and attention, the orienting task also modulates organization effects by encouraging participants to 
elaborate on certain dimensions of the stimuli. For example, with an orienting task that emphasized the emotional nature of the stimuli both emotion and relatedness improved memory additively, so that related neutral words were recalled to the same extent as unrelated emotional words, both of which were less well recalled than related emotional words; but with a familiarity orienting task controlling organization abolished EEM (Ferré et al., 2014).

One literature that cares very much about the semantic cohesiveness of stimuli is the literature on false memory. Extensive research shows that participants can produce false memories for items that are semantically related to stimuli presented during an encoding task. The Deese-Roediger-McDermott (DRM) paradigm, presents participants with a list of words that are all associated with a nonpresented critical lure (Deese, 1959; Roediger and McDermott, 1995). To illustrate, the words bed, dream, and nap may be presented at encoding but not the critical lure sleep, which can be falsely recognized or recalled to the same extent as true memories (Stadler et al., 1999), with high confidence and a distinctive sense of recollection (Roediger and McDermott, 1995). In the emotional variant of the DRM (Moritz et al., 2005), each word list is associated with a critical lure that is either positive (holidays), depressionrelevant (loneliness), or neutral (window). Whether emotional valence and arousal influence false memory rates in the emotional DRM paradigm is not yet established (Brueckner and Moritz, 2009; Corson and Verrier, 2007; Moritz et al., 2005; Stea et al., 2013). However, participants report more false emotional memories and recollection ('remember') judgments that are congruent with their mood, and specific discrete emotions, at encoding (Bland et al., 2016; Knott and Thorley, 2014; Ruci et al., 2009). This may result from spreading activation from the concepts activated during encoding to neighboring concepts including the critical lure (associative activation theory; Howe et al., 2009). Within this framework, emotion serves to improve the conceptual links across items and to provide a unifying way to group them together (Gallo et al., 2009). Alternatively, as the verbatim memory trace fades, dependence on gist memory traces increases (fuzzy-trace theory; Brainerd and Reyna, 2002). The spreading activation or increased reliance on gist will lead participants to erroneously recall or recognize any concept that is emotionally- or semantically-related to those that were experienced during encoding. 
As discussed in more detail in Section 4.3, free recall relies greatly on memory search, and is therefore fairly sensitive to organization effects. The magnitude of the EEM will therefore depend in part on whether the semantic cohesiveness of neutral stimuli was controlled, so that it resembled the semantic cohesiveness of emotional stimuli (Buchanan et al., 2006; Talmi et al., 2007b; Talmi and Moscovitch, 2004); the greatest EEM will be found when emotional stimuli are compared to unselected neutral stimuli. The semantic links between emotional stimuli also lead to semantic clustering around emotional categories in free recall (Long et al., 2015; Talmi et al., 2007a). In recognition memory tests, the greater semantic cohesiveness of emotional stimuli changes bias, encouraging participants to be more lenient in endorsing emotional stimuli as old, resulting in an increased hit rate but also false alarm rate (Dougal and Rotello, 2007). The bias can be avoided by matching the level of organization of emotional and neutral stimuli. When semantic relatedness is controlled, there is no evidence for increased false alarms for emotional pictures (Choi et al., 2013). Consideration of discrete emotions may also be important as hits and false alarm rates can differ across discrete emotions, such as disgust, fear, and sadness (Marchewka et al., 2016).

\subsection{Emotional Stimuli are Distinctive}

Emotional stimuli stand out relative to neutral stimuli because they have unique attributes not shared by neutral stimuli (Schmidt, 1991). For example, compared to a picture of a child walking to school, a picture of an injured child has unique objective features (e.g. facial expression) and because it triggers feelings and unique cognitive processes (e.g. reflexive attention and appraisal of goal-relevance). Schmidt suggests that emotional stimuli are distinct in both an 'absolute' and a 'relative' sense, compared to other items in long-term and working memory, respectively. For example, a picture of a woman wearing a dress is not absolutely distinct, but it would be distinct relative to a set of pictures of nude models (Schmidt, 2002).

Distinctive items naturally capture encoding resources, but only relative distinctiveness reliably enhances memory (Schmidt, 2002, 1991). For example, memory for a picture of a clothed model was better than memory for a picture of a nude model when both were presented mixed within a set of nude models (Schmidt, 2002). Relative distinctiveness can capture attention at encoding, but even when it does not, it can enhance the competitive advantage of distinct stimuli at retrieval. Thus, for example, when common and bizarre sentences were studied 
together, bizarre sentences were only remembered better than common sentences when participants were asked to recall them together, but not when they were recalled separately (McDaniel et al., 2005). In the emotional memory literature Dewhurst and Parry (2000) first demonstrated that primary distinctiveness modulates the EEM by manipulating list composition in a remember-know recognition paradigm. In their study, positive and negative words were remembered better than neutral words when they were all presented together in lists of 'mixed' valence, but not when words of each valence were presented in separate 'pure' lists. Therefore, even though emotional words were always distinct in an 'absolute' sense, this did not produce a memory advantage in the pure lists, suggesting that only relative distinctiveness contributes to the EEM. This conclusion was supported by several studies where EEM in free recall was stronger when items were studied and recalled together, than when they were studied and recalled separately (Hadley and MacKay, 2006; Schmidt and Saari, 2007; Talmi et al., 2007a; Talmi and McGarry, 2012).

\subsection{Joint Contribution of Cognitive Factors to Immediate EEM}

Attentional focus, the ease of organization, and the processing of similarities and differences between emotional and neutral stimuli all contribute to increase the EEM effect separately, but in many experiments they can work together. In fact, the typical paradigm in many human experiments encourages this joint contribution by using incidental encoding instructions where participants have no particular reason to attend to neutral stimuli, unselected neutral stimuli that are likely less cohesive, and mixed lists where emotional items would be distinct both at encoding and retrieval. A few studies illustrate this joint contribution. Schmidt and Saari (2007) showed, for example, that taboo words attracted attention and were consequently remembered better regardless of list composition, whereas non-taboo emotional words did not attract more attention than neutral words in mixed list designs, but were still recalled better than neutral words in these designs, an effect attributed to their increased distinctiveness. It follows, therefore, that greater experimental control over differences between emotional and neutral stimuli should weaken the EEM. 


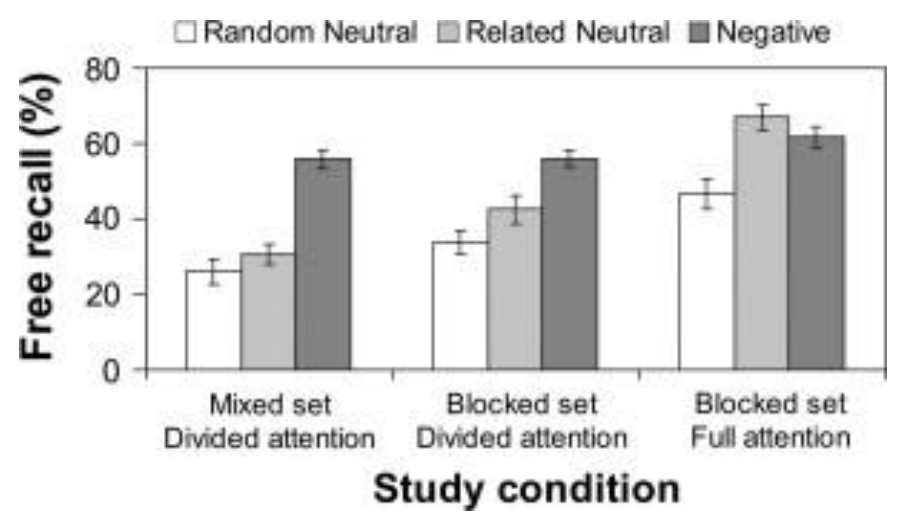

Figure 9. Immediate free recall of negative, semantically-related neutral, and random neutral pictures in full- and divided-attention conditions manipulating set composition (mixed vs. blocked sets). Immediate EEM was largest in the divided-attention mixed condition and abolished in the full-attention blocked condition that controlled for the effects of attention, relatedness, and relative distinctiveness. Reprinted from Talmi, D., McGarry, L.M., 2012. Accounting for immediate emotional memory enhancement. J. Mem. Lang. 66, 93-108, with permission.

Experimental control matters more when the methodology allows the differences between emotional and neutral stimulus processing to influence memory. Free recall tests are least constrained; Talmi and colleagues have found that they needed to control at least three factors to abolish immediate EEM in free recall: attention, organization, and relative distinctiveness (Talmi and McGarry, 2012; see Figure 9). The experimental set-up can be chosen, therefore, to minimize influences of cognitive factors and increase the likelihood of abolishing immediate EEM. Sharot and Phelps (2004), for example, presented stimuli briefly and at the periphery, and used forced-choice recognition memory tests. They did not observe EEM immediately, but it appeared in delayed tests, possibly as a result of modulated consolidation. The interesting ways that emotional stimuli are processed differently at encoding could, in principle, have greater impact on delayed memory. If possible, these effects should be controlled before we can be certain that delayed EEM stems from arousal-dependent modulated consolidation.

\section{Focal Enhancements of Memory}

One of the open questions in the emotional memory literature is the degree to which emotional effects on memory are selective, on the one hand, or 'spill over' to increase or decrease memory for neighboring stimuli. Animal work is beginning to be able to address this question by moving 
from tasks like spatial learning and one-trial avoidance learning to NOR. In the human literature the question relates to whether emotion changes memory for the emotional stimulus itself, or also for its associates and source context, and whether these effects extend spatially or temporally to other stimuli that are presented close to the emotional stimulus.

\subsection{Spatial Neighbors}

Attending to emotional stimuli can affect the processing of neighbors in space. According to the attentional narrowing hypothesis (Easterbrook, 1959), central details of an arousing event are processed at the expense of non-emotional peripheral details (also referred to as memory tradeoff effect; Christianson, 1992; Levine and Edelstein, 2009; Phelps, 2012; Reisberg and Heuer, 2003). This phenomenon is commonly observed in witnesses to a crime, who show increased attentional focus on a criminal's weapon at the expense of processing the rest of the crime scene ('weapon focus' effect; Loftus et al., 1987; for a meta-analysis, see Fawcett et al., 2013). But what determines which features in a scene will be preferentially processed relative to others? Many distinctions have been proposed although they all agree that emotion produces focal enhancements in memory rather than improves overall memory for all aspects of an event. The first distinction is between 'central' and 'peripheral' information that can be defined in one of three ways: information that an emotionally-aroused person would or should attend to (i.e., 'attention magnets'); information that is spatially, temporally, or conceptually central to an emotional stimulus; or information that is related to a person's currently active goals (Levine and Edelstein, 2009). A second distinction is based on the extent to which features are bound during encoding, with arousal increasing the binding of constituent (within-item) features of emotional stimuli, whereas between-item details are not bound by arousal (Mather, 2007). A third distinction is made between intrinsic item features and extrinsic contextual features, with arousal enhancing memory for the former but not the latter (Kensinger, 2009). Regardless of the operational definition employed, the following sections will discuss findings of emotion-induced focal enhancements of memory.

As reviewed in Section 3.1, arousing stimuli tend to capture more attention resources than less-arousing stimuli. It can be expected, then, that as more attention is dedicated to processing features of an arousing stimulus, fewer resources will be available to process other features of the event. In a review on the focal enhancements of memory, Kensinger (2009) 
revealed that emotion produced the strongest focal enhancements when 'attention magnets' (i.e., items that 'grab' attention) were present in a scene and when the encoding task encouraged participants to focus on the affective aspects of a scene compared to the visual details. In a series of experiments, Kensinger and colleagues examined the encoding processes responsible for focal memory effects using the 'same-similar-new' recognition memory task. In this task, participants are shown emotional and neutral ('central') objects on a neutral ('peripheral') background; for example, a snake (negative) or monkey (neutral) on a jungle background (neutral). At test, participants view objects and backgrounds separately and are instructed to identify which are: the exact same as the studied items, similar in verbal label to the studied items but different in visual details, or new objects and backgrounds not previously studied (for an illustration of the task, see Figure 10). In this task, correct same responses measure a participant's ability to remember the exact visual details of an item. Using the subsequent-memory paradigm, Kensinger et al. (2007a) found that the successful recognition of same negative objects correlated with amygdala and fusiform gyrus (a structure involved in high-level visual processing) activation at encoding. However, amygdala activity at encoding did not predict memory for the encoding task (animacy or size decisions made about the objects), suggesting that the amygdala is selectively involved in enhancing memory for features central to the emotional object (i.e., its visual features), especially those that increase memory vividness (Kensinger et al., 2011), but not all contextual details (i.e., encoding task decisions; Kensinger et al., 2007a). Other structures involved in the affective attentional network (orbitofrontal cortex, anterior cingulate gyrus, ventral striatum) were involved in increasing memory for visual details of negative objects while decreasing memory for contextual details (Kensinger, 2009). The increased activity in the affective attentional network may ensure that the intrinsic features of negative items are successfully encoded, leading more extrinsic features of the event to be forgotten (Kensinger, 2009). This should not be confused with contextual 'source' information related to the presentation of an emotional stimulus (e.g., font color, spatial location) that may perhaps improve with emotion (for a review, see Kensinger and Schacter, in press). 

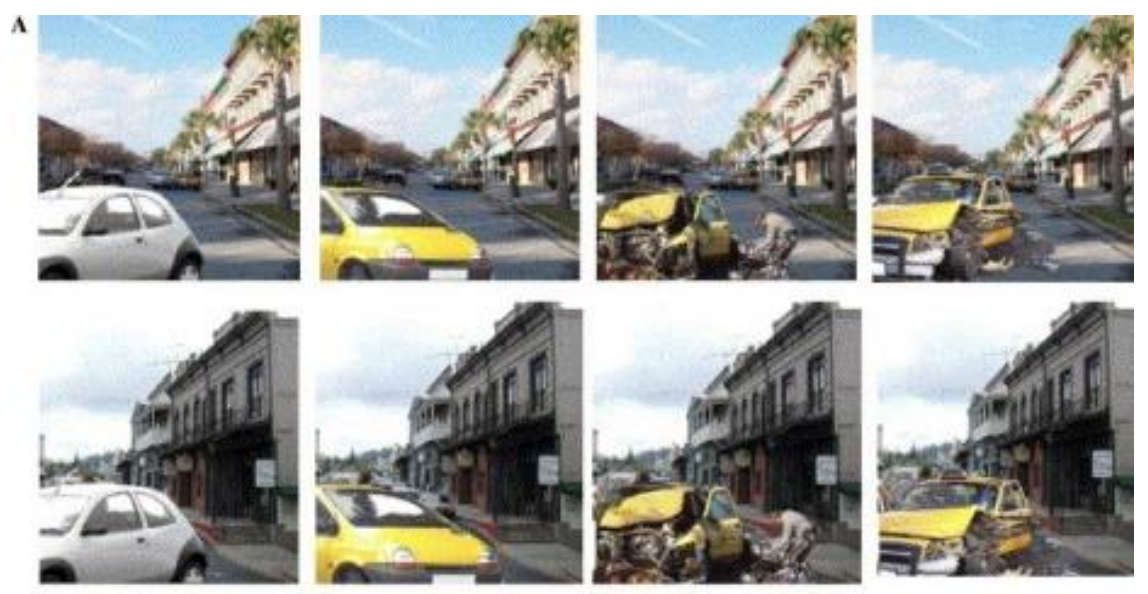

B Did you see a taxi cab accident?
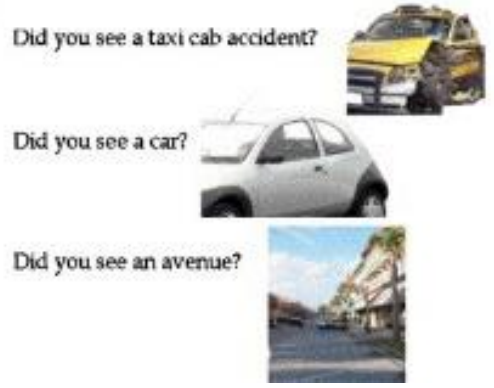

Figure 10. In the same-similar-new paradigm, a negative or neutral ('central') object is presented in front of a neutral ('peripheral') background. There are eight versions for each scene so as to vary the details of the objects and backgrounds (A). After viewing the scenes, participants are shown objects and backgrounds and instructed to identify which are: the exact same as the studied items, similar in verbal label but different in visual detail, or new items not previously studied (B). Reprinted from Kensinger, E.A., Garoff-Eaton, R.J., Schacter, D.L., 2007b. Effects of emotion on memory specificity: Memory trade-offs elicited by negative visually arousing stimuli. J. Mem. Lang. 56, 575-591, with permission.

The same-similar-new recognition task also allows for a comparison between memory for visual details (same responses) and memory for the 'gist' or meaning of the event (calculated from similar and new responses), in addition to dissociating memory for 'central' objects and 'peripheral' backgrounds. Kensinger et al. (2007b) reported a robust emotion-induced memory trade-off for central and peripheral information but not for gist and visual details. Enhanced memory for central emotional objects impaired memory for non-emotional background information. While emotion did not result in differences in gist and visual detail memory for objects, memory for gist exceeded memory for visual details for non-emotional backgrounds presented with emotional objects. That is, memory for non-emotional background information was impaired when presented with an emotional object compared to a neutral object, an 
impairment that was particularly pronounced for detail memory than for gist memory. These results appear to contradict classic findings of emotion enhancing the memory for gist at the expense of memory for detail (Adolphs et al., 2005, 2001), although these studies did not clearly differentiate between central and peripheral information but rather considered gist to relate to the meaning of an event relative to low-arousing and unimportant background details. Some researchers examine differences in gist and detail only for the central objects (e.g., Xu et al., 2011) to eliminate this confound and have found similar findings of increased memory for details of emotional objects relative to neutral ones. Arousal-biased competition theory (discussed in Section 3.1) offers a way to reconcile discrepancies in this literature, by suggesting that the fate of central/peripheral and gist/detail information is determined by the priority it receives during encoding - it is processed well if it is deemed important, but otherwise suppressed regardless if it is central or related to the gist of the event (Mather and Sutherland, 2011). According to this theory, stimuli that receive higher priority during encoding will be dedicated more processing resources.

In addition, memory for detail may differ for positive and negative information. Although both lead to EEM effects and are supported by an emotional memory network (LaBar and Cabeza, 2006), negative information appears to be remembered in greater visual detail than positive information. Negative events may specifically draw and 'lock' more spatial attention than neutral or positive events, as evidenced by decreased activity in the intraparietal cortex, an area that helps to shift attention in visual space (Pourtois and Vuilleumier, 2006). Using the same-similar-new paradigm, Kensinger and Schacter (2008) found that participants had a better memory for the visual details of negative objects (as measured by an increased ability to discriminate same and similar objects) than they did for positive or neutral objects. In addition, the successful encoding of negative objects depended on increased activity in the visual cortex and fusiform gyrus (both related to sensory processing) whereas the successful encoding of positive objects depended on increased activity in the lateral prefrontal and temporal regions (both related conceptual or semantic processing). Similar findings (Mickley and Kensinger, 2008) were demonstrated using the remember-know task whereby remember responses (episodic recollection) for negative information related to greater temporo-occipital recruitment (associated with sensory processing) during encoding, whereas positive information that was 'known' (remembered with limited episodic details) related to greater recruitment of the 
cingulate gyrus, and frontal and parietal areas, more involved in conceptual processing. Some evidence suggests that these valence-dependent networks are also present during retrieval as well (for a review, see Kensinger, 2009). Together these studies suggest that negative information is remembered in great visual detail whereas the gist of positive information is more likely to be remembered than its specific details and that these processes are supported by valence-dependent networks during encoding and retrieval. This is congruent with findings that central-peripheral memory trade-offs are more robust for negative than for positive stimuli, as negative information appears to narrow attention to central details whereas positive information widens attention to both central and peripheral information (Berntsen, 2002; Chipchase and Chapman, 2013; Libkuman et al., 2004; Talarico et al., 2009). Therefore, researchers must carefully dissociate memory trade-offs for central-peripheral and gist-detail information, as negative stimuli produce robust enhancements for visually-detailed, central objects, whereas these distinctions may be less relevant for positive stimuli.

\subsection{Temporal Neighbors: Anterograde and Retrograde Amnesia}

What is the fate of neighbors in time to that of emotional items or events? The memory consequences of temporal attention modulation have been explored in list settings, examining the negative (but sometimes positive) carryover effects that emotional stimuli produce on memory for their neutral neighbors in time. Negative carryover effects can reduce memory for neutral items presented before (Hurlemann et al., 2005; Mackay et al., 2004; Strange et al., 2003) or after (Mackay et al., 2004; Mathewson et al., 2008; Schmidt, 2002) an emotional stimulus (termed retrograde and anterograde amnesia, respectively). The negative carryover effects may result in part from more resources being dedicated to the contextual binding (Hadley and MacKay, 2006; Mackay et al., 2004; Mather, 2007) and maintenance in working memory (Sutherland and Mather, 2012) of emotional stimuli at the expense of temporally neighboring neutral stimuli. As emotional stimuli receive higher priority during encoding, fewer resources are available to process temporal neighbors (Mather and Sutherland, 2011). As a result, negative carryover effects are more robust when resources at encoding are limited by task demands (e.g., during the rapid presentation of stimuli; Hadley and MacKay, 2006), leaving even fewer resources available for low-priority items. In contrast, in a few studies memory was enhanced for neutral items presented before an arousing stimulus or event (Anderson et al., 2006; Dunsmoor et 
al., 2015), although there is some evidence that positive carryover effects only occur on delayed memory tests (Anderson et al., 2006; Dunsmoor et al., 2015; Knight and Mather, 2009). This may result from the influence of emotional arousal on the relatively-weak memory for neutral stimuli presented in the same context.

In a recent six-experiment study, Schmidt and Schmidt (2016) provided an exhaustive account of the effects of temporal proximity on early long-term memory for highly arousing taboo words, less arousing non-taboo emotional words, and semantically-related neutral words. First, there were no positive carryover effects reported in any of the experiments, perhaps due to brief test delays (3 minutes or less). Second, higher arousal (taboo words) was associated with larger and more reliable negative carryover effects than lower arousal (emotional words). Third, anterograde amnesia appeared under a variety of conditions but retrograde amnesia was observed in more limited conditions. The results suggested that anterograde amnesia is related to the attentional capture of emotional stimuli, whereas retrograde amnesia is influenced at least in part by retrieval processes. It would be instructive to repeat this work using EEG, to explore the relationship between the memory effects and the time course of emotional modulation of attention. Moreover, EEG studies suggest that ERP amplitude for emotional scenes is modulated by emotion for up to $6 \mathrm{~s}$ (Schupp et al., 2006).

\subsection{Recollection versus Familiarity}

As mentioned in Section 1.1, episodic memories are vivid, detailed recollections of previously-experienced events. It is reasonably well-accepted that when episodic memories are 'truly episodic' they involve recollection, which can be distinguished from familiarity (a more general sense that one has encountered this particular stimulus before). Recollection clearly depends heavily on the medial temporal lobe, and different structures within the medial temporal lobe (e.g., hippocampus proper vs. adjacent perirhinal cortex) may contribute differentially to recollection and familiarity (Brown and Aggleton, 2001; Eichenbaum et al., 2007; Squire et al., 2007; Wixted and Squire, 2010). Beyond the MTL, prefrontal and parietal cortex and other structures (Skinner and Fernandes, 2007) also support recollection.

We are beginning to understand how these integrated brain networks supporting recollection and familiarity interact with emotion. Focusing on recollection, Murty et al. (2010) conducted a meta-analysis of the first generation of single-trial fMRI studies of memory and 
episodic memory encoding. Delays between study and test ranged from a few minutes to several weeks. Of note, most of these studies tested memory using recognition rather than recall (see below) but many of the recognition tests assessed recollection. Emotional encoding effects were consistently found in the medial temporal lobe (amygdala, hippocampus, parahippocampal gyrus) and, in keeping with Skinner and Fernandes' (2007) broader view of the brain bases of recollection, emotional encoding effects were also found in prefrontal and parietal regions. Murty and colleagues argued that these activation patterns were commensurate with the guiding of attention by emotion that we have outlined in Section 3.1. Note however, that emotional recollection fMRI effects are not isolated to encoding: Dolcos et al. (2005) examined recollection of emotional pictures compared to neutral ones in a recognition memory task with a 1-year delay. Recollection of emotional items was associated with greater activity in the amygdala and the medial temporal lobe than for familiar or forgotten emotional items. This study, among others, has emphasized greater co-activation of amygdala, hippocampus, and connected regions as playing a crucial role in the enhancement of episodic memory by emotion over time (Fink et al., 1996; Ritchey et al., 2008; Smith et al., 2006).

One of the most straightforward ways to assess recollection is a first-person subjective report from the participant on whether they felt they could 'remember' or merely 'know' that they had encountered the target in the study list previously. This method was employed by the majority of the neuroimaging studies mentioned in the previous paragraph. It draws on Tulving and colleagues' (Tulving, 1985; Wheeler et al., 1997) emphasis on a subjective sense of 'mental time travel' accompanying the retrieval of an episodic memory. In classic behavioral applications of this method to emotional episodic memory, Ochsner (2000) and Kensinger and Corkin (2003) reported that emotional pictures and words led to an enhanced subjective sense of 'remembering,' and little if any effect on familiarity, on a later memory test. Myriad other studies have replicated this finding (Sharot et al., 2004; Yonelinas and Ritchey, 2015).

Alternatively, one can gauge recollection and familiarity by contrasting results from tests of free recall (a method that is supposed to tap recollection more heavily than familiarity) compared to those from standard methods of recognition (a method that may be supported more equally by recollection or familiarity). The EEM is probably more robust when assessed using free recall than when using recognition. This is arguably in part because free recall engages more 
controlled processing during retrieval than does recognition (Craik and Lockhart, 1972; Craik and McDowd, 1987). Because free recall requires participants to actively retrieve information from memory, participants will be more likely to retrieve emotional information because of the processing advantages at encoding, consolidation, and retrieval. On the other hand, in an old-new recognition task, stimuli are presented for a second time and participants must simply decide whether each stimulus matches a representation in memory. Providing that neutral stimuli were at least superficially processed at encoding, they should later be recognized to a similar extent as emotional stimuli. Despite this clear hypothesis, few studies have examined it properly, namely, by eliminating possible order effects by using between-subject designs, whereby participants are randomly assigned to complete either the free recall or recognition test. What little work there is has shown an advantage of free recall over recognition when testing memory for a slideshow after one week (Brust and Stein, 2012) but no advantage when testing memory for words immediately after encoding (Aycicegi and Harris, 2004). Therefore, more studies are needed to determine whether the advantage of recall over recognition is time- or stimulus-dependent.

Conclusions about the selectivity of the emotional effect on recollection depend critically on how recollection is defined. For some researchers the ability to retain the precise details of the previous experience is essential, which might lead to the use of two-alternative forced choice testing using original versus mirror-reversed versions of a target (Touryan et al., 2007). For others, who would operationalize recollection as being best indicated by the ability to bind disparate bits of information together into an episode, associative memory paradigms such as verbal paired-associate recall may be key (Bisby and Burgess, 2014; Madan et al., 2012). For those who emphasize the binding of items and their context source monitoring paradigms may be preferred, where the match between an item and its original presentation context are identified by participants, but note that the context can be spatial, temporal, or perceptual. Across these literatures each using a different operational definition of 'recollection' it is often difficult to discern clear patterns - there is nothing like the consistency that is seen with the Remember/Know paradigm. Even within each literature, across different studies using very similar methods, there is often surprising lack of consistency (contrast findings using similar source memory paradigms from Davidson et al., 2006; Doerksen and Shimamura, 2001). In evaluating which paradigm is most suitable it is important to consider whether the 'target' and 'contextual' information are presented in the same place and/or at the same time (see Sections 
4.1 and 4.2), whether the 'target' and 'contextual' information are emotional versus neutral and whether one tests associative memory using recall or recognition (Madan et al., 2012), uses positively- or negatively-valenced items, or uses a relatively short or long delay between study and test (Pierce and Kensinger, 2011). In many paradigms, and in paired-associate recall paradigms in particular, it now seems essential to tease apart emotion's effects on attention to each of the items in a pair at encoding (e.g., an emotional item might draw attention away from the neutral associate with which it is paired; see Section 3.1) and to differentiate between effects of emotion on memory for each of the items in a pair versus memory for the association between those items specifically (Bisby and Burgess, 2014; Madan et al., 2012; see also Bisby et al., 2016). A final crucial consideration is how easily to to-be-linked information can be 'unitized' into a single representation (Chiu et al., 2013; Murray and Kensinger, 2013). One key recent development is the examination of multiple operational definitions of 'recollection' within the same paradigm: An emerging possibility is that emotion enhances subjective recollection (as assessed via Remember/Know self-report), but does not enhance, or might even impair, memory for associated contextual detail (Bisby and Burgess, 2014; Kensinger et al., 2007a; Madan et al., 2012; Sharot and Yonelinas, 2008; for a review, see Yonelinas and Ritchey, 2015).

\section{Methodological Considerations}

Up to now we have emphasized the relative consistency in findings on the EEM across various materials and paradigms. In this practical section we review factors that inform the selection of material and the sample.

\subsection{What Is Being Remembered?}

\subsubsection{Experimenter-generated stimuli}

Almost all human studies compare a set of stimuli that come with pre-existing emotional associations against a set of stimuli that have minimal emotional associations. Selection of the emotional stimuli requires care. For example, the previous sections have made it clear that arousal is thought to play a pivotal role in the EEM. Many studies of the effects of arousal use both negative and positive stimuli, so that arousal effects are not confounded by those of valence. From a practical perspective, however, many authors have reported difficulty in equating the arousal level of positive and negative stimuli, a complication that creates its own interpretative 
problem. Additionally, the most arousing positive visual stimuli are sexual images, which researchers may be reluctant to use or which could have gender-specific effects, and cartoons, which may require more prolonged elaboration before their meaning is appreciated. Valence could also exert effects on memory by inducing differential processing at encoding. Kensinger and colleagues have pointed out that valenced stimuli may be higher in semantic cohesiveness, and hypothesized that participants may be more likely to elaborate on valenced, compared to neutral words, even when neither are arousing (holiday compared to message; Kensinger, 2004). This hypothesis was supported by findings that subsequent-memory effects for positive stimuli rely more on activation in frontal brain regions (Mickley Steinmetz and Kensinger, 2009). Valence also influenced effective connectivity between the amygdala and other brain regions (Mickley Steinmetz et al., 2010). These same challenges occur in selection of the non-emotional stimuli for comparison. That is, this approach obviously runs the risk of introducing confounds if the different sets differ from one another in other (i.e., non-emotional) ways. Further, each type of material brings specific considerations.

Words are relatively well-understood by cognitive psychologists, and are relatively easy to select to reduce the influence of confounding factors involving the words themselves (e.g., length, frequency, imageability) and the lists into which they are assembled (e.g., distinctiveness, semantic inter-relatedness). Importantly, even the most emotionally evocative words ('decapitation') still produce relatively modest subjective and physiological arousal. Given that arousal is a key factor in EEM, this is a serious limitation. To overcome it researchers can use taboo words, which are thought to be more emotionally salient. The EEM has been demonstrated with taboo words even when it is absent with semantically-related emotional words (Buchanan et al., 2006; Madan et al., 2016). The trouble, of course, is that it is more difficult to control taboo words for confounding factors. In an elegant item-based analysis Madan and colleagues showed that the property of 'tabooness' explained free recall better than other emotional properties of the words, including arousal and the time spent on the orienting task. Future work may be able to determine what psychological processes explain EEM for taboo words.

It is easier to find highly-arousing emotional pictures (Lang et al., 2008) than emotional words (Bradley and Lang, 1999), although participants' ratings of arousal for pictures today may be lower than in the past (Betella and Verschure, 2016; Libkuman et al., 2007). Pictures might 
also be used more easily to trigger specific emotions such as sadness, disgust, and fear to study the effects of discrete emotions on memory (Chapman et al., 2013, 2009). It may, however, be more difficult to minimize confounds with pictures than words because most picture sets provide additional ratings on only a few non-emotional factors. Several standardized picture sets exist; without question, the International Affective Picture System (IAPS; Lang et al., 2008) is the most popular, whereas the Nencki Affective Picture System (NAPS; Marchewka et al., 2014) currently provides the most additional information, including on some visual properties and semantic category membership.

Film clips contain movement, so they are more realistic and lifelike than static pictures. Movement is a powerful cue to object identity and to attention: Moving images are 'behaviorally urgent' and capture attention (Franconeri and Simons, 2003) and boost physiological arousal more easily than do static images (Detenber and Simons, 1998; Simons et al., 2003). Perhaps for these reasons, moving images are often more emotionally powerful than static images (Courtney et al., 2010). Given these advantages, it is surprising that relatively few emotional clip sets have been developed (e.g., Bos et al., 2013; Carvalho et al., 2012; Fernández et al., 2012). The sets that do exist typically include only a small number of clips, usually from well-known feature films (Gabert-Quillen et al., 2015; Matthews et al., 2010; Schaefer et al., 2010). These clips' familiarity can influence participants' emotional responses, eye movements, attention, and memory. As an alternative, recently we have collected a set of almost 300 brief emotional film clips (Tanguay et al., 2016). These are novel to most viewers, to minimize confounding effects of familiarity, and can be used in designs similar to those for static pictures and words.

Slideshows and stories arguably are life-like while allowing for tighter experimental control than film clips. Slideshows ideally take the same series of pictures and alter some of them so that one version is imbued with emotion, whereas the other version minimizes emotion. Often the two versions have the same neutral content at the beginning of the story but then reach a point of divergence between emotional and neutral versions part-way through. The best-known example comes from Cahill and McGaugh (1995), a story of a mother and child visiting the father's workplace. An emotional or a neutral narrative is played over the slides to color participants` interpretation of what they are seeing: In the emotional version, the child is hit by a car en route. Other materials following a similar logic have also been developed (Leal et al., 
2016). Available stimuli of these nature are, however, often limited to one neutral version and one emotional version (although for a recent innovation, see Kim et al., 2013).

\subsubsection{Autobiographical memory}

Emotional autobiographical memories for personal events are elicited with a word cue (knife) or event cue (Tell me about your wedding day), whereas memories for emotional public events are often cued with a brief description or a date (How did you hear about September 11, 2001?). Autobiographical memories (especially emotional ones) play significant roles in our lives - they contribute to the sense of self, regulate emotions, create and maintain social ties, and help us teach and inform others - that laboratory tasks have difficulty matching (Bluck, 2003; see Chapter 12). While such stimuli are high in ecological validity, they force researchers to relinquish experimental control: Events may follow a similar script but differ in terms of many details from one person to another, and delays between the event and the memory report can vary across participants or be difficult to ascertain. Accuracy of memory reports can rarely be guaranteed, leading researchers to rely on factors such as recollective experience, number of details in the memory report, or consistency of repeated within-subject memory reports (with the idea that if two separate reports on the same event from the same person are different over time, at least one of the reports is inaccurate) as proxies for accuracy. As a clever solution to the problem of inter-individual variability (e.g., one person's wedding compared to another's), some researchers have asked people about the same event, but taken care to select an event that is viewed as positive by some respondents and negative by others (e.g., asking fans of two opposing sports teams about the game they played, or supporters of two rival politicians about a debate between the two; Bohn and Berntsen, 2007; Breslin and Safer, 2011; Hastorf and Cantril, 1954; Kensinger and Schacter, 2006b). This type of approach provides a relatively tight control, and has revealed generally more similarities than differences between positively and negativelytinged memories of the same event.

Considering these complications, it is perhaps remarkable that a clear picture of emotion's influence on memory in the real world is emerging: Similar to the laboratory, emotional memories are often more detailed and strong than neutral ones. Strongly negative experiences can lead to vivid, long-lasting memories: In one of the few studies able to verify 
memory reports objectively for accuracy, McKinnon et al. (2015) found that passengers aboard a trans-Atlantic flight retained clear, detailed memories of their near-crash into the ocean.

Are there clear differences in emotional autobiographical memory based on valence? It has been argued that positive memories tend to be strengthened and emphasized over time whereas negative ones are weakened and de-emphasized, possibly via controlled retrieval and motivated forgetting. This 'Pollyanna principle' (Matlin and Stang, 1978) and the related phenomenon of the fading affect bias, in which the strength of emotions associated with negative autobiographical memories fades more rapidly than it does for positive ones (Walker and Skowronski, 2009) have been argued to be psychologically protective, not only to keep one's emotional life pleasant (Kappes and Crockett, 2016; Kouchaki and Gino, 2016; Richard et al., 2003; Ritchie et al., 2006) but also to guide current and future behavior. The data so far, however, have been mixed at best on this question. Similar to laboratory studies, for autobiographical memories valence may be a less important factor than arousal (Talarico et al., 2004; compare with Bradley et al., 1992).

\subsection{Who Is Remembering?}

It goes without saying that individual differences are common among participants in ratings of emotional stimuli. For example, even if an image of a shark earns a mean rating of 3/10 on a Likert-type rating scale from $1=$ extremely negative to $10=$ extremely positive, some participants who are very fond of sharks will give a rating of 10 . An easy way to accommodate for this in later memory analyses is to only include trials as 'emotional' for each participant based on his or her individual ratings, but this will lead to differences in the numbers of trials assigned to each participant, which has its own disadvantages.

Perhaps more importantly, interactions between participant characteristics and stimuli may have significant but underappreciated effects on memory. These characteristics include participants' sex, which might influence emotional brain responses and behavioral memory patterns. Women and men show somewhat different neuroimaging correlates of emotional memory encoding (early studies emphasized sex differences in lateralization of emotion-related responses; Cahill et al., 2004; Canli et al., 2002a) although the reliability and functional implications of these potential differences remain unclear. Women sometimes show a greater emotional enhancement of memory than men (e.g., Canli et al., 2002a), perhaps in part because 
women can find negative stimuli to be more psychologically and/or physiologically arousing than men do (Bradley et al., 2001; Segal and Cahill, 2009). Cahill and colleagues have focused on sex hormones as a key reason for differences between men's and women's brain responses and emotional memory patterns. These researchers have further suggested that women's emotional memory varies across the menstrual cycle (Nielsen et al., 2013a) and that taking oral contraceptives (which often include high doses of estrogen and progestin) can blunt women's stress responses but enhance their memory for the gist of an emotional experience (Nielsen et al., 2013b, 2011). The field may need to consider more carefully the effects of sex differences and sex hormones in research design and analyses (Sakaki and Mather, 2012).

Other potentially influential individual characteristics include personality traits. Neuroticism and extraversion are the two that have been studied most extensively in relation to emotion, but there is still surprisingly little work on their connection to memory specifically. The existing evidence suggests that people who are more extraverted tend to be more reactive to positive arousing stimuli, and those who are more neurotic tend to be more attuned to negative arousing stimuli information (Canli et al., 2002b, 2001; Kehoe et al., 2012). Here it should be noted that some of these studies (e.g., Canli et al., 2001; Kehoe et al., 2012) have included only young women, with no information on their menstrual cycles or their oral contraceptive use. Given the potential influence of these factors outlined in the paragraph above, caution is warranted in interpreting this literature. Even if individual differences in these trait differences in emotion processing are reliable, only a handful of studies have examined their potential ramifications for memory (Bradley and Mogg, 1994; Denkova et al., 2012).

One possibility is that the reason people with certain personality traits differ in their memory for emotional stimuli is that they encode these stimuli differently to begin with. Personality traits do seem to affect attention (e.g., Amin et al., 2004; Derryberry and Reed, 1994; Reed and Derryberry, 1995), but, given the sometimes lack of connection between emotion effects on attention and those on encoding (Section 3.1) this question requires further research (for a summary of the methodological considerations discussed in this chapter, see Figure 11). 


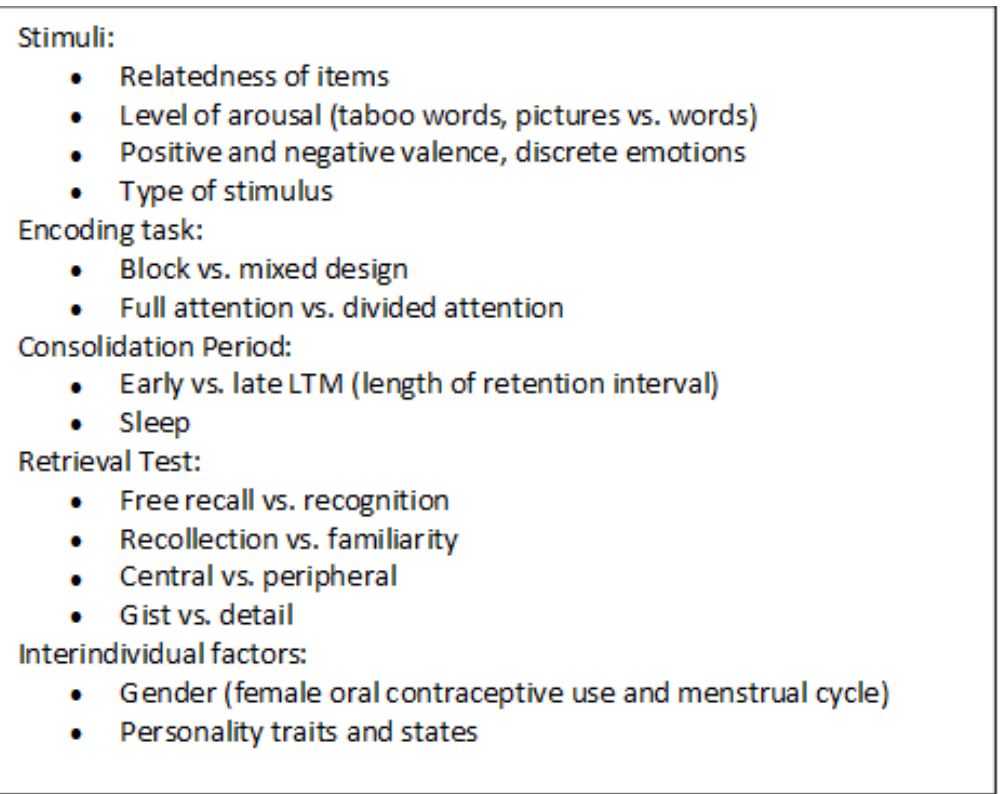

Figure 11. Summary of methodological considerations for studies of EEM.

\section{Final Remarks}

As we have seen throughout this review, as a field we have made much progress on answering questions about how emotion and episodic memory interact. In this final section we will present three emerging research directions. The first concerns the operationalization of emotional arousal in the laboratory. The reliance on pre-experimental differences between emotional and neutral stimuli (Section 5.1, e.g., taboo versus neutral words) comes at the cost of removing an essential experimental control. Manipulating emotion via inherent stimulus differences run the risk of introducing confounds if the different sets differ from one another in other ways - and there is no way of guaranteeing against this. If we continue to use such stimuli because of their value in evoking true emotional experiences we must, as a community, invest more in understanding how they differ from each other. For example, what aspect(s) of the emotional pictures makes participants rate them as more related to each other (Chao et al., 2015)? Could non-emotional factors of visual 'salience' be controlled better (Parkhurst et al., 2002)?

Alternatively, a promising but under-used strategy to exert experimental control is to select symbols that are objectively neutral (e.g., abstract shapes), and conditioning some to become positive, some to become negative, and others to remain neutral. This would allow researchers to counterbalance which stimuli were associated with which specific emotions, and 
thus claim more confidently that one is seeing effects of emotion per se on memory, and not of other, confounding factors (e.g. Lee et al., 2014). Another alternative is to manipulate the goals of participants, for example, to examine memory for game-relevant stimuli (Montagrin et al., 2013) or memory for food in hungry participants (Talmi et al., 2013). We may sense here an influence of the way researchers conceptualize emotion. Using pre-experimentally emotional stimuli is common in the literature that supports dimensional views of emotion, whereas the other two methodologies draw, respectively, on learning and appraisal perspectives on emotion.

The second research direction is an attempt to better integrate animal and human research (an example of the challenges in bridging the gap is seen in Section 2.4), in order to capitalize better on the research potential afforded in animal models, for example in the development of psychiatric drugs (LeDoux and Pine, 2016). One strategy is to bring animal research more in line with human research. This includes using 'list-like' stimulus material, for instance NOR rather than one-trial avoidance learning, and memory tasks that are episodic-like, rather than those more closely linked with non-declarative memory. The 'what-where-when' paradigm is promising in this regard (Eacott and Easton, 2010). In it, different aspects of context can be examined separately, or memory for the conjunction of these different elements can be probed. While this paradigm has rarely been used with intense emotion, it arguably provides a more rigorous test of episodic (or episodic-like) memory in non-humans.

Coming from the other direction, it would be equally useful to bring human work on emotional memory more in line with existing animal work, by examining memory for simpler, but personally meaningful, rewarding or punishing stimuli and contexts. This agenda also touches on a rational analysis of emotional memory, asking 'what EEM is good for'. This question is difficult to ask with the tasks and stimuli currently prevalent in the emotional memory literature, because we cannot ever really know when it is, or is not, evolutionary advantageous to remember emotionally-evocative experiences. It is more feasible to rationally analyze tasks where animals and humans have an opportunity to use memory to optimize decision-making (Doll et al., 2015). So long as humans and non-humans solve these tasks using similar cognitive and neural systems (Holland and Smulders, 2011), comparative conclusions will become more persuasive. 
And finally, experimental work on human emotional memory has been inspired by the very many interesting questions that emotional memories pose, and by their great potential to illuminate societal and psychological phenomena such as eye-witness testimony, flash-bulb memories and aberrant memories in mental health disorders. There has been relatively less focus on theory development. Most existing theories of emotional memory emphasize the implementation level (Cahill and McGaugh, 1998; Mather et al., 2015; Yonelinas and Ritchey, 2015, but see Mather, 2007; Mather and Sutherland, 2011; Talmi, 2013) more than the algorithmic or computational levels of analysis. Indeed, the emotional memory literature has developed somewhat independently from the mainstream cognitive literature on human memory. As reiterated above, emotion is complex, and has many cognitive implications; this is exactly the kind of situation where cognitive modelling could be useful. Much progress can be made in understanding emotional memory by asking how emotion modulates known parameters of existing memory models, for example the Context Maintenance and Retrieval model (Long et al., 2015; Polyn et al., 2009) or the Complementary Learning Systems (O'Reilly et al., 2014).

As we noted at the outset, the zeitgeist regarding emotion and memory has evolved over time. Today, we arguably find ourselves caught between two zeitgeists (at least, in Western thinking): On the one hand, emotions are thought of as primitive and animalistic, diametrically opposed to the human capacity for reason. On the other hand, emotions are understood to mark us as fully human, as opposed to cold, calculating machines. Our review of the literature makes it clear that modern research on emotional memory has gone beyond these widespread worldviews. By embracing the potential advantages of comparative research, deeper analysis of the subtleties of emotion and of memory, and adaptation of advancing technology we will better be able to understand why we remember so well those emotional moments from our lives that make each of us who we are. 


\section{References}

Abercrombie, H., Speck, N., Monticelli, R., 2006. Endogenous cortisol elevations are related to memory facilitation only in individuals who are emotionally aroused.

Psychoneuroendocrinology 31, 187-196. doi:10.1016/j.psyneuen.2005.06.008

Adolphs, R., Cahill, L., Schul, R., Babinsky, R., 1997. Impaired declarative memory for emotional material following bilateral amygdala damage in humans. Learn. Mem. 4, 291-300. doi:10.1101//m.4.3.291

Adolphs, R., Denburg, N., Tranel, D., 2001. The Amygdala's Role in Long-Term Declarative Memory for Gist and Detail. Behav. Neurosci. 115, 983-992. doi:10.1037/07357044.115.5.983

Adolphs, R., Tranel, D., Buchanan, T.W., 2005. Amygdala damage impairs emotional memory for gist but not details of complex stimuli. Nat. Neurosci. 8, 512-518. doi:10.1038/nn1413

Alba, J., W., Hasher, L., 1983. Is memory schematic? Psychol. Bull. 93, 203-231.

Algom, D., Chajut, E., Lev, S., 2004. A Rational Look at the Emotional Stroop Phenomenon: A Generic Slowdown, Not a Stroop Effect. J. Exp. Psychol. Gen. 133, 323-338. doi:10.1037/0096-3445.133.3.323

Amin, Z., Todd Constable, R., Canli, T., 2004. Attentional bias for valenced stimuli as a function of personality in the dot-probe task. J. Res. Personal., Proceedings of the Association for Research in Personality 38, 15-23. doi:10.1016/j.jrp.2003.09.011

Anderson, A.K., 2005. Affective Influences on the Attentional Dynamics Supporting Awareness. J. Exp. Psychol. Gen. 134, 258-281. doi:10.1037/0096-3445.134.2.258

Anderson, A.K., Wais, P.E., Gabrieli, J.D., 2006. Emotion enhances remembrance of neutral events past. Proc. Natl. Acad. Sci. 103, 1599-1604.

Armitage, R., 2007. Sleep and circadian rhythms in mood disorders. Acta Psychiatr. Scand. Suppl. 104-115. doi:10.1111/j.1600-0447.2007.00968.x

Arnold, M.B., 1960. Emotion and personality. Columbia University Press, New York, NY.

Atienza, M., Cantero, J.L., 2008. Modulatory effects of emotion and sleep on recollection and familiarity. J. Sleep Res. 17, 285-294. doi:10.1111/j.1365-2869.2008.00661.x

Averill, J., 1980. A constructionist view of emotion, in: Plutchik, R., Kellerman, H. (Eds.), Emotion: Theory, Research, and Experience. Academic Press, New York, NY.

Aycicegi, A., Harris, C., 2004. BRIEF REPORT Bilinguals' recall and recognition of emotion words. Cogn. Emot. 18, 977-987. doi:10.1080/02699930341000301

Barrett, L.F., 2014. The conceptual act theory: A précis. Emot. Rev. 6, 292-297.

Barrett, L.F., Russell, J.A. (Eds.), 2015. The psychological construction of emotion. Guilford Press, New York, NY.

Bass, D.I., Nizam, Z.G., Partain, K.N., Wang, A., Manns, J.R., 2014. Amygdala-mediated enhancement of memory for specific events depends on the hippocampus. Neurobiol. Learn. Mem. 107, 37-41. doi:10.1016/j.nlm.2013.10.020

Bass, D.I., Partain, K.N., Manns, J.R., 2012. Event-specific enhancement of memory via brief electrical stimulation to the basolateral complex of the amygdala in rats. Behav. Neurosci. 126, 204-208. doi:10.1037/a0026462

Baumeister, R.F., Bratslavsky, E., Finkenauer, C., Vohs, K.D., 2001. Bad is stronger than good. Rev. Gen. Psychol. 5, 323-370. doi:10.1037//1089-2680.5.4.323 
Bennion, K.A., Mickley Steinmetz, K.R., Kensinger, E.A., Payne, J.D., 2015. Sleep and cortisol interact to support memory consolidation. Cereb. Cortex N. Y. N 1991 25, 646-657. doi:10.1093/cercor/bht 255

Berntsen, D., 2002. Tunnel memories for autobiographical events: Central details are remembered more frequently from shocking than from happy experiences. Mem. Cognit. 30, 1010-1020.

Berridge, K.C., Robinson, T.E., Aldridge, J.W., 2009. Dissecting components of reward: "liking", "wanting", and learning. Curr. Opin. Pharmacol. 9, 65-73. doi:10.1016/j.coph.2008.12.014

Betella, A., Verschure, P.F.M.J., 2016. The Affective Slider: A Digital Self-Assessment Scale for the Measurement of Human Emotions. PLOS ONE 11, e0148037. doi:10.1371/journal.pone.0148037

Bianchin, M., Mello-e-Souza, T., Medina, J.H., Izquierdo, I., 1999. The amygdala is involved in the modulation of long-term memory but not in working or short-term memory. Neurobiol. Learn. Mem. 71, 127-131.

Bisby, J.A., Burgess, N., 2014. Negative affect impairs associative memory but not item memory. Learn. Mem. 21, 21-27. doi:10.1101/lm.032409.113

Bisby, J.A., Horner, A.J., Hørlyck, L.D., Burgess, N., 2016. Opposing effects of negative emotion on amygdalar and hippocampal memory for items and associations. Soc. Cogn. Affect. Neurosci. 11, 981-990. doi:10.1093/scan/nsw028

Bland, C.E., Howe, M.L., Knott, L., 2016. Discrete emotion-congruent false memories in the DRM paradigm. Emotion 16, 611-619. doi:10.1037/emo0000153

Bluck, S., 2003. Autobiographical memory: exploring its functions in everyday life. Memory 11, 113-123. doi:10.1080/741938206

Bohn, A., Berntsen, D., 2007. Pleasantness bias in flashbulb memories: Positive and negative flashbulb memories of the fall of the Berlin Wall among East and West Germans. Mem. Cognit. 35, 565-577. doi:10.3758/BF03193295

Boiger, M., Mesquita, B., 2012. The construction of emotion in interactions, relationships, and cultures. Emot. Rev. 4, 221-229.

Bos, M.G.N., Jentgens, P., Beckers, T., Kindt, M., 2013. Psychophysiological Response Patterns to Affective Film Stimuli. PLOS ONE 8, e62661. doi:10.1371/journal.pone.0062661

Bradley, B.P., Mogg, K., 1994. Mood and personality in recall of positive and negative information. Behav. Res. Ther. 32, 137-141.

Bradley, M.M., Codispoti, M., Sabatinelli, D., Lang, P.J., 2001. Emotion and motivation II: sex differences in picture processing. Emotion 1, 300-319.

Bradley, M.M., Greenwald, M.K., Petry, M.C., Lang, P.J., 1992. Remembering pictures: pleasure and arousal in memory. J. Exp. Psychol. Learn. Mem. Cogn. 18, 379-390.

Bradley, M.M., Lang, P.J., 1999. Affective norms for English words (ANEW): Instruction manual and affective ratings. Technical report $\mathrm{C}-1$, the center for research in psychophysiology, University of Florida.

Brainerd, C.J., Reyna, V.F., 2002. Fuzzy-trace theory and false memory. Curr. Dir. Psychol. Sci. $11,164-169$.

Breslin, C.W., Safer, M.A., 2011. Effects of Event Valence on Long-Term Memory for Two Baseball Championship Games. Psychol. Sci. 956797611419171. doi:10.1177/0956797611419171 
Brown, M.W., Aggleton, J.P., 2001. Recognition memory: what are the roles of the perirhinal cortex and hippocampus? Nat. Rev. Neurosci. 2, 51-61.

Brueckner, K., Moritz, S., 2009. Emotional valence and semantic relatedness differentially influence false recognition in mild cognitive impairment, Alzheimer's disease, and healthy elderly. J. Int. Neuropsychol. Soc. 15, 268. doi:10.1017/S135561770909047X

Brust, P.G., Stein, L.M., 2012. Memory for emotional events: The role of time of testing and type of test. Univ. Psychol. 11, 79-90.

Buchanan, T.W., Etzel, J.A., Adolphs, R., Tranel, D., 2006. The influence of autonomic arousal and semantic relatedness on memory for emotional words. Int. J. Psychophysiol. 61, 2633. doi:10.1016/j.ijpsycho.2005.10.022

Cahill, L., Alkire, M.T., 2003. Epinephrine enhancement of human memory consolidation: interaction with arousal at encoding. Neurobiol. Learn. Mem. 79, 194-198.

Cahill, L., Babinsky, R., Markowitsch, H., J., McGaugh, J.L., 1995. The amygdala and emotional memory. Nature 377, 295-296.

Cahill, L., Gorski, L., Le, K., 2003. Enhanced human memory consolidation with post-learning stress: interaction with the degree of arousal at encoding. Learn. Mem. 10, 270-274.

Cahill, L., McGaugh, J.L., 1998. Mechanisms of emotional arousal and lasting declarative memory. Trends Neurosci. 21, 294-299.

Cahill, L., McGaugh, J.L., 1995. A novel demonstration of enhanced memory associated with emotional arousal. Conscious. Cogn. 4, 410-421.

Cahill, L., Prins, B., Weber, M., McGaugh, J.L., 1994. Beta-Adrenergic activation and memory for emotional events. Lett. Nat. 371, 702-704.

Cahill, L., Uncapher, M., Kilpatrick, L., Alkire, M.T., Turner, J., 2004. Sex-Related Hemispheric Lateralization of Amygdala Function in Emotionally Influenced Memory: An fMRI Investigation. Learn. Mem. 11, 261-266. doi:10.1101/1m.70504

Canli, T., Desmond, J.E., Zhao, Z., Gabrieli, J.D., 2002a. Sex differences in the neural basis of emotional memories. Proc. Natl. Acad. Sci. 99, 10789-10794.

Canli, T., Sivers, H., Whitfield, S.L., Gotlib, I.H., E, D., 2002b. Amygdala response to happy faces as a function of extraversion. Science 296, 2191. doi:10.1126/science.1068749

Canli, T., Zhao, Z., Desmond, J.E., Kang, E., Gross, J., Gabrieli, J.D., 2001. An fMRI study of personality influences on brain reactivity to emotional stimuli. Behav. Neurosci. 115, 3342.

Cape, E.G., Jones, B.E., 1998. Differential modulation of high-frequency gammaelectroencephalogram activity and sleep-wake state by noradrenaline and serotonin microinjections into the region of cholinergic basalis neurons. J. Neurosci. Off. J. Soc. Neurosci. 18, 2653-2666.

Carvalho, S., Leite, J., Galdo-Álvarez, S., Gonçalves, O.F., 2012. The Emotional Movie Database (EMDB): a self-report and psychophysiological study. Appl. Psychophysiol. Biofeedback 37, 279-294. doi:10.1007/s10484-012-9201-6

Chao, Y.-W., Wang, Z., Mihalcea, R., Deng, J., 2015. Mining semantic affordances of visual object categories, in: Proceedings of the IEEE Conference on Computer Vision and Pattern Recognition. pp. 4259-4267.

Chapman, H.A., Johannes, K., Poppenk, J.L., Moscovitch, M., Anderson, A.K., 2013. Evidence for the differential salience of disgust and fear in episodic memory. J. Exp. Psychol. Gen. 142, 1100-1112. doi:10.1037/a0030503 
Chapman, H.A., Kim, D.A., Susskind, J.M., Anderson, A.K., 2009. In Bad Taste: Evidence for the Oral Origins of Moral Disgust. Science 323, 1222-1226.

doi:10.1126/science. 1165565

Cheeta, S., Ruigt, G., van Proosdij, J., Willner, P., 1997. Changes in sleep architecture following chronic mild stress. Biol. Psychiatry 41, 419-427. doi:10.1016/S0006-3223(96)00058-3

Chipchase, S.Y., Chapman, P., 2013. Trade-offs in visual attention and the enhancement of memory specificity for positive and negative emotional stimuli. Q. J. Exp. Psychol. 66, 277-298. doi:10.1080/17470218.2012.707664

Chiu, Y.-C., Dolcos, F., Gonsalves, B.D., Cohen, N.J., 2013. On opposing effects of emotion on contextual or relational memory. Emot. Sci. 103. doi:10.3389/fpsyg.2013.00103

Choi, H.-Y., Kensinger, E.A., Rajaram, S., 2013. Emotional content enhances true but not false memory for categorized stimuli. Mem. Cognit. 41, 403-415. doi:10.3758/s13421-0120269-2

Christianson, S.-Å., 1992. Emotional stress and eyewitness memory: A critical review. Psychol. Bull. 112, 284-309.

Chun, M.M., Golomb, J.D., Turk-Browne, N.B., 2011. A Taxonomy of External and Internal Attention. Annu. Rev. Psychol. 62, 73-101. doi:10.1146/annurev.psych.093008.100427

Conway, M.A., 2009. Episodic memories. Neuropsychologia, Episodic Memory and the Brain 47, 2305-2313. doi:10.1016/j.neuropsychologia.2009.02.003

Corbetta, M., Shulman, G.L., 2002. Control of Goal-Directed and Stimulus-Driven Attention in the Brain. Nat. Rev. Neurosci. 3, 215-229. doi:10.1038/nrn755

Corson, Y., Verrier, N., 2007. Emotions and False Memories. Psychol. Sci. 18, 208-211. doi:10.1111/j.1467-9280.2007.01874.X

Courtney, C.G., Dawson, M.E., Schell, A.M., Iyer, A., Parsons, T.D., 2010. Better than the real thing: eliciting fear with moving and static computer-generated stimuli. Int. J.

Psychophysiol. Off. J. Int. Organ. Psychophysiol. 78, 107-114. doi:10.1016/j.ijpsycho.2010.06.028

Craik, F.I., Lockhart, R.S., 1972. Levels of processing: A framework for memory research. J. Verbal Learn. Verbal Behav. 11, 671-684.

Craik, F.I., McDowd, J.M., 1987. Age differences in recall and recognition. J. Exp. Psychol. Learn. Mem. Cogn. 13, 474-479. doi:10.1037/0278-7393.13.3.474

Craik, F.I.M., Blankstein, K.R., 1975. Psychophysiology and human memory. Res. Psychophysiol. 388-417.

Damasio, A.R., Everitt, B.J., Bishop, D., 1996. The Somatic Marker Hypothesis and the Possible Functions of the Prefrontal Cortex [and Discussion]. Philos. Trans. R. Soc. Lond. B Biol. Sci. 351, 1413-1420. doi:10.1098/rstb.1996.0125

Davidson, P.S.R., McFarland, C.P., Glisky, E.L., 2006. Effects of emotion on item and source memory in young and older adults. Cogn. Affect. Behav. Neurosci. 6, 306-322.

Davis, M., Whalen, P.J., 2001. The amygdala: vigilance and emotion. Mol. Psychiatry 6, 13-34.

De Boer, S.F., Koopmans, S.J., Slangen, J.L., Van der Gugten, J., 1990. Plasma catecholamine, corticosterone and glucose responses to repeated stress in rats: effect of interstressor interval length. Physiol. Behav. 47, 1117-1124.

Deese, J., 1959. On the prediction of occurrence of particular verbal intrusions in immediate recall. J. Exp. Psychol. 58, 17-22.

Deliens, G., Neu, D., Peigneux, P., 2013. Rapid eye movement sleep does not seem to unbind memories from their emotional context. J. Sleep Res. 22, 656-662. doi:10.1111/jsr.12065 
Denkova, E., Dolcos, S., Dolcos, F., 2012. Reliving emotional personal memories: affective biases linked to personality and sex-related differences. Emot. Wash. DC 12, 515-528. doi:10.1037/a0026809

Derryberry, D., Reed, M.A., 1994. Temperament and attention: orienting toward and away from positive and negative signals. J. Pers. Soc. Psychol. 66, 1128-1139.

Detenber, B.H., Simons, R.F., 1998. Roll 'em!: The effects of picture motion on emotional responses. J. Broadcast. Electron. Media 42, 113.

Dewhurst, S.A., Parry, L.A., 2000. Emotionality, distinctiveness, and recollective experience. Eur. J. Cogn. Psychol. 12, 541-551.

D’Hooge, R., De Deyn, P.P., 2001. Applications of the Morris water maze in the study of learning and memory. Brain Res. Rev. 36, 60-90.

Diekelmann, S., Born, J., 2010. The memory function of sleep. Nat. Rev. Neurosci. 11, 114-126. doi: $10.1038 / \mathrm{nrn} 2762$

Doerksen, S., Shimamura, A.P., 2001. Source memory enhancement for emotional words. Emot. Wash. DC 1, 5-11.

Dolcos, F., LaBar, K.S., Cabeza, R., 2005. Remembering one year later: role of the amygdala and the medial temporal lobe memory system in retrieving emotional memories. Proc. Natl. Acad. Sci. U. S. A. 102, 2626-2631.

Dolcos, F., LaBar, K.S., Cabeza, R., 2004a. Interaction between the amygdala and the medial temporal lobe memory system predicts better memory for emotional events. Neuron 42 , 855-863.

Dolcos, F., LaBar, K.S., Cabeza, R., 2004b. Dissociable effects of arousal and valence on prefrontal activity indexing emotional evaluation and subsequent memory: an eventrelated fMRI study. NeuroImage 23, 64-74. doi:10.1016/j.neuroimage.2004.05.015

Doll, B.B., Shohamy, D., Daw, N.D., 2015. Multiple memory systems as substrates for multiple decision systems. Neurobiol. Learn. Mem. 117, 4-13. doi:10.1016/j.nlm.2014.04.014

Dornelles, A., de Lima, M.N.M., Grazziotin, M., Presti-Torres, J., Garcia, V.A., Scalco, F.S., Roesler, R., Schroder, N., 2007. Adrenergic enhancement of consolidation of object recognition memory. Neurobiol. Learn. Mem. 88, 137-142.

doi:10.1016/j.nlm.2007.01.005

Dougal, S., Rotello, C.M., 2007. "Remembering" emotional words is based on response bias, not recollection. Psychon. Bull. Rev. 14, 423-429.

Dudai, Y., 2004. The Neurobiology of Consolidations, Or, How Stable is the Engram? Annu. Rev. Psychol. 55, 51-86. doi:10.1146/annurev.psych.55.090902.142050

Dunsmoor, J.E., Murty, V.P., Davachi, L., Phelps, E.A., 2015. Emotional learning selectively and retroactively strengthens memories for related events. Nature 520, 345-348. doi:10.1038/nature14106

Eacott, M.J., Easton, A., 2010. Episodic memory in animals: Remembering which occasion. Neuropsychologia, Animal Models of Amnesia 48, 2273-2280. doi:10.1016/j.neuropsychologia.2009.11.002

Easterbrook, J.A., 1959. The effect of emotion on cue utilization and the organization of behavior. Psychol. Rev. 66, 183-201.

Eich, E., Geraerts, E., Schooler, J.W., Forgas, J.P., 2008. Memory in and about Affect, in: Learning and Memory: A Comprehensive Reference. Academic Press, pp. 239-260. 
Eichenbaum, H., Yonelinas, A.R., Ranganath, C., 2007. The Medial Temporal Lobe and Recognition Memory. Annu. Rev. Neurosci. 30, 123-152. doi:10.1146/annurev.neuro.30.051606.094328

Einstein, G.O., Hunt, R.R., 1980. Levels of processing and organization: Additive effects of individual-item and relational processing. J. Exp. Psychol. [Hum. Learn.] 6, 588.

Ekman, P., 1992. An argument for basic emotions. Cogn. Emot. 6, 169-200.

Ekman, P., Friesen, W.V., Ellsworth, P., 1972. Emotion in the human face: Guidelines for research and an integration of findings. Pergamon, New York, NY.

Eysenck, M.W., 1976. Arousal, learning, and memory. Psychol. Bull. 83, 389-404.

Fawcett, J.M., Russell, E.J., Peace, K.A., Christie, J., 2013. Of guns and geese: a meta-analytic review of the "weapon focus" literature. Psychol. Crime Law 19, 35-66. doi:10.1080/1068316X.2011.599325

Fernández, C., Pascual, J.C., Soler, J., Elices, M., Portella, M.J., Fernández-Abascal, E., 2012. Physiological responses induced by emotion-eliciting films. Appl. Psychophysiol. Biofeedback 37, 73-79. doi:10.1007/s10484-012-9180-7

Ferré, P., Fraga, I., Comesaña, M., Sánchez-Casas, R., 2014. Memory for emotional words: The role of semantic relatedness, encoding task and affective valence. Cogn. Emot. 0, 1-10. doi:10.1080/02699931.2014.982515

Fink, G.R., Markowitsch, H.J., Reinkemeier, M., Bruckbauer, T., Kessler, J., Heiss, W.D., 1996. Cerebral representation of one's own past: neural networks involved in autobiographical memory. J. Neurosci. Off. J. Soc. Neurosci. 16, 4275-4282.

Folarin, B.A., 1981. Is Grouping of Words in Memory a Fast or a Slow Process? Psychol. Rep. 48, 355-358. doi:10.2466/pr0.1981.48.2.355

Fox, E., Russo, R., Dutton, K., 2002. Attentional Bias for Threat: Evidence for Delayed Disengagement from Emotional Faces. Cogn. Emot. 16, 355-379.

Franconeri, S.L., Simons, D.J., 2003. Moving and looming stimuli capture attention. Percept. Psychophys. 65, 999-1010. doi:10.3758/BF03194829

Frey, S., Bergado-Rosado, J., Seidenbecher, T., Pape, H.-C., Frey, J.U., 2001. Reinforcement of early long-term potentiation (early-LTP) in dentate gyrus by stimulation of the basolateral amygdala: heterosynaptic induction mechanisms of late-LTP. J. Neurosci. 21, 3697-3703.

Frey, U., Morris, R.G., 1998. Synaptic tagging: implications for late maintenance of hippocampal long-term potentiation. Trends Neurosci. 21, 181-188.

Gabert-Quillen, C.A., Bartolini, E.E., Abravanel, B.T., Sanislow, C.A., 2015. Ratings for emotion film clips. Behav. Res. Methods 47, 773-787. doi:10.3758/s13428-014-0500-0

Galliot, E., Levaillant, M., Beard, E., Millot, J.-L., Pourié, G., 2010. Enhancement of spatial learning by predator odor in mice: involvement of amygdala and hippocampus. Neurobiol. Learn. Mem. 93, 196-202. doi:10.1016/j.nlm.2009.09.011

Gallo, D.A., Foster, K.T., Johnson, E.L., 2009. Elevated false recollection of emotional pictures in young and older adults. Psychol. Aging 24, 981-988. doi:10.1037/a0017545

Gershman, S.J., Daw, N.D., In press. Reinforcement Learning and Episodic Memory in Humans and Animals: An Integrative Framework. Annu. Rev. Psychol. 68. doi:10.1146/annurevpsych-122414-033625

Giedke, H., Schwärzler, F., 2002. Therapeutic use of sleep deprivation in depression. Sleep Med. Rev. 6, 361-377. 
Gold, P.E., Edwards, R.M., McGaugh, J.L., 1975a. Amnesia produced by unilateral, subseizure, electrical stimulation of the amygdala in rats. Behav. Biol. 15, 95-105.

Gold, P.E., Hankins, L., Edwards, R.M., Chester, J., McGaugh, J.L., 1975b. Memory interference and facilitation with posttrial amygdala stimulation: Effect on memory varies with footshock level. Brain Res. 86, 509-513.

Gold, P.E., van Buskirk, R., 1978. Posttraining brain norepinephrine concentrations: Correlation with retention performance of avoidance training and with peripheral epinephrine modulation of memory processing. Behav. Biol. 23, 509-520. doi:10.1016/S00916773(78)91614-0

Gottesmann, C., Gottesman, I., 2007. The neurobiological characteristics of rapid eye movement (REM) sleep are candidate endophenotypes of depression, schizophrenia, mental retardation and dementia. Prog. Neurobiol. 81, 237-250.

doi:10.1016/j.pneurobio.2007.01.004

Gülçay, Ç., Cangöz, B., 2016. Effects of emotion and perspective on remembering events: An eye-tracking study. J. Eye Mov. Res. 9. doi:10.16910/jemr.9.2.4

Hadley, C.B., MacKay, D.G., 2006. Does Emotion Help or Hinder Immediate Memory? Arousal Versus Priority-Binding Mechanisms. J. Exp. Psychol. Learn. Mem. Cogn. 32, 79-88. doi:10.1037/0278-7393.32.1.79

Hamann, S., 2001. Cognitive and neural mechanisms of emotional memory. Trends Cogn. Sci. 5, 394-400.

Hamann, S.B., Cahill, L., McGaugh, J.L., Squire, L.R., 1997. Intact enhancement of declarative memory for emotional material in amnesia. Learn. Mem. 4, 301-309. doi:10.1101/1m.4.3.301

Hamann, S.B., Cahill, L., Squire, L.R., 1997. Emotional perception and memory in amnesia. Neuropsychology 11, 104-113. doi:10.1037/0894-4105.11.1.104

Hastorf, A.H., Cantril, H., 1954. They saw a game; a case study. J. Abnorm. Soc. Psychol. 49, 129-134. doi: 10.1037/h0057880

Holland, S.M., Smulders, T.V., 2011. Do humans use episodic memory to solve a What-WhereWhen memory task? Anim. Cogn. 14, 95-102. doi:10.1007/s10071-010-0346-5

Houwer, J.D., Hermans, D., 1994. Differences in the affective processing of words and pictures. Cogn. Emot. 8, 1-20. doi:10.1080/02699939408408925

Howe, M.L., Wimmer, M.C., Gagnon, N., Plumpton, S., 2009. An associative-activation theory of children's and adults' memory illusions. J. Mem. Lang. 60, 229-251. doi:10.1016/j.jml.2008.10.002

Hu, P., Stylos-Allan, M., Walker, M.P., 2006. Sleep facilitates consolidation of emotional declarative memory. Psychol. Sci. 17, 891-898. doi:10.1111/j.1467-9280.2006.01799.x

Humphreys, L., Underwood, G., Chapman, P., 2010. Enhanced memory for emotional pictures: A product of increased attention to affective stimuli? Eur. J. Cogn. Psychol. 22, 12351247. doi:10.1080/09541440903427487

Hunt, R.R., Mcdaniel, M.A., 1993. The Enigma of Organization and Distinctiveness. J. Mem. Lang. 32, 421-445. doi:10.1006/jmla.1993.1023

Hurlemann, R., Hawellek, B., Matusch, A., Kolsch, H., Wollersen, H., Madea, B., Vogeley, K., Maier, W., Dolan, R.J., 2005. Noradrenergic Modulation of Emotion-Induced Forgetting and Remembering. J. Neurosci. 25, 6343-6349. doi:10.1523/JNEUROSCI.0228-05.2005

Izard, C.E., 2011. Forms and Functions of Emotions: Matters of Emotion-Cognition Interactions. Emot. Rev. 3, 371-378. doi:10.1177/1754073911410737 
Izard, C.E., 2010. The many meanings/aspects of emotion: definitions, functions, activation, and regulation. Emot. Rev. 2, 363-370.

James, W., 1890. The Principles of Psychology. Henry Holt and Company, New York, NY.

Joëls, M., Pu, Z., Wiegert, O., Oitzl, M.S., Krugers, H.J., 2006. Learning under stress: how does it work? Trends Cogn. Sci. 10, 152-158. doi:10.1016/j.tics.2006.02.002

Kahana, M.J., Howard, M.W., Polyn, S.M., 2008. Associative retrieval processes in episodic memory.

Kandel, E.R., Dudai, Y., Mayford, M.R., 2014. The Molecular and Systems Biology of Memory. Cell 157, 163-186. doi:10.1016/j.cell.2014.03.001

Kaplan, R., Kaplan, S., 1969. The arousal-retention interval interaction revisited: The effects of some procedural changes. Psychon. Sci. 15, 84-85.

Kaplan, S., Kaplan, R., 1968. Arousal and memory: A comment. Psychon. Sci. 10, 291-292.

Kappes, A., Crockett, M.J., 2016. The Benefits and Costs of a Rose-Colored Hindsight. Trends Cogn. Sci. 20, 644-646. doi:10.1016/j.tics.2016.06.009

Kehoe, E.G., Toomey, J.M., Balsters, J.H., Bokde, A.L.W., 2012. Personality modulates the effects of emotional arousal and valence on brain activation. Soc. Cogn. Affect. Neurosci. 7, 858-870. doi:10.1093/scan/nsr059

Kensinger, E.A., 2009. Remembering the Details: Effects of Emotion. Emot. Rev. 1, 99-113. doi: $10.1177 / 1754073908100432$

Kensinger, E.A., 2008. Age differences in memory for arousing and nonarousing emotional words. J. Gerontol. B. Psychol. Sci. Soc. Sci. 63, P13-P18.

Kensinger, E.A., 2004. Remembering emotional experiences: The contribution of valence and arousal. Rev. Neurosci. 15, 241-251.

Kensinger, E.A., Addis, D.R., Atapattu, R.K., 2011. Amygdala activity at encoding corresponds with memory vividness and with memory for select episodic details. Neuropsychologia 49, 663-673. doi:10.1016/j.neuropsychologia.2011.01.017

Kensinger, E.A., Corkin, S., 2004. Two routes to emotional memory: Distinct neural processes for valence and arousal. Proc. Natl. Acad. Sci. U. S. A. 101, 3310-3315.

Kensinger, E.A., Corkin, S., 2003. Memory enhancement for emotional words: Are emotional words more vividly remembered than neutral words? Mem. Cognit. 31, 1169-1180.

Kensinger, E.A., Garoff-Eaton, R.J., Schacter, D.L., 2007a. How negative emotion enhances the visual specificity of a memory. J. Cogn. Neurosci. 19, 1872-1887.

Kensinger, E.A., Garoff-Eaton, R.J., Schacter, D.L., 2007b. Effects of emotion on memory specificity: Memory trade-offs elicited by negative visually arousing stimuli. J. Mem. Lang. 56, 575-591. doi:10.1016/j.jml.2006.05.004

Kensinger, E.A., Schacter, D.L., 2008. Neural Processes Supporting Young and Older Adults' Emotional Memories. J. Cogn. Neurosci. 20, 1161-1173.

Kensinger, E.A., Schacter, D.L., 2006a. Processing emotional pictures and words: Effects of valence and arousal. Cogn. Affect. Behav. Neurosci. 6, 110-126.

Kensinger, E.A., Schacter, D.L., 2006b. When the Red Sox shocked the Yankees: Comparing negative and positive memories. Psychon. Bull. Rev. 13, 757-763. doi:10.3758/BF03193993

Kensinger, E.A., Schacter, D.L., in press. Memory and emotion, in: Lewis, M., Haviland-Jones, J.M., Barrett, L.F. (Eds.), Handbook of Emotion. Guilford Press, New York City, USA.

Kern, R.P., Libkuman, T.M., Otani, H., Holmes, K., 2005. Emotional Stimuli, Divided Attention, and Memory. Emotion 5, 408-417. doi:10.1037/1528-3542.5.4.408 
Kilpatrick, L., 2003. Amygdala modulation of parahippocampal and frontal regions during emotionally influenced memory storage. NeuroImage 20, 2091-2099.

doi:10.1016/j.neuroimage.2003.08.006

Kim, J.S.-C., Vossel, G., Gamer, M., 2013. Effects of Emotional Context on Memory for Details: The Role of Attention. PLOS ONE 8, e77405. doi:10.1371/journal.pone.0077405

Kleinsmith, L.J., Kaplan, S., 1964. Interaction of arousal and recall interval in nonsense syllable paired-associate learning. J. Exp. Psychol. 67, 124-126. doi:10.1037/h0045203

Kleinsmith, L.J., Kaplan, S., 1963. Paired-associate learning as a function of arousal and interpolated interval. J. Exp. Psychol. 65, 190.

Klumpers, F., Morgan, B., Terburg, D., Stein, D.J., van Honk, J., 2015. Impaired acquisition of classically conditioned fear-potentiated startle reflexes in humans with focal bilateral basolateral amygdala damage. Soc. Cogn. Affect. Neurosci. 10, 1161-1168. doi:10.1093/scan/nsu164

Knight, M., Mather, M., 2009. Reconciling findings of emotion-induced memory enhancement and impairment of preceding items. Emotion 9, 763-781.

Knott, L.M., Thorley, C., 2014. Mood-congruent false memories persist over time. Cogn. Emot. 28, 903-912. doi:10.1080/02699931.2013.860016

Koch, A., Alves, H., Krüger, T., Unkelbach, C., 2016. A general valence asymmetry in similarity: Good is more alike than bad. J. Exp. Psychol. Learn. Mem. Cogn. 42, 11711192. doi:10.1037/xlm0000243

Koriat, A., Pansky, A., Goldsmith, M., 2011. An output-bound perspective on false memories: The case of the Deese-Roediger-McDermott (DRM) paradigm, in: Benjamin, A.S., Bjork, R.A. (Eds.), Successful Remembering and Successful Forgetting: A Festschrift in Honor of Robert A. Bjork. Psychology Press, New York, NY.

Kouchaki, M., Gino, F., 2016. Memories of unethical actions become obfuscated over time. Proc. Natl. Acad. Sci. 113, 6166-6171. doi:10.1073/pnas.1523586113

Kouider, S., Eger, E., Dolan, R., Henson, R.N., 2009. Activity in face-responsive brain regions is modulated by invisible, attended faces: evidence from masked priming. Cereb. Cortex N. Y. N 1991 19, 13-23. doi:10.1093/cercor/bhn048

LaBar, K.S., Cabeza, R., 2006. Cognitive neuroscience of emotional memory. Nat. Rev. Neurosci. 7, 54-64. doi:10.1038/nrn1825

LaBar, K.S., Cabeza, R., 2006. Cognitive neuroscience of emotional memory. Nat. Rev. Neurosci. 7, 54-64. doi:10.1038/nrn1825

LaBar, K.S., Phelps, E.A., 1998. Arousal-Mediated Memory Consolidation: Role of the Medial Temporal Lobe in Humans. Psychol. Sci. 9, 490-493. doi:10.1111/1467-9280.00090

Lang, P.J., Bradley, M.M., Cuthbert, B.N., 2008. International affective picture system (IAPS): Affective ratings of pictures and instruction manual, in: Technical Report A-8. University of Florida, Gainesville, FL.

Lazarus, R.S., 1991. Emotion and adaptation. Oxford University Press, New York, NY.

Leal, S.L., Noche, J.A., Murray, E.A., Yassa, M.A., 2017. Age-related individual variability in memory performance is associated with amygdala-hippocampal circuit function and emotional pattern separation. Neurobiol. Aging 49, 9-19. doi:10.1016/j.neurobiolaging.2016.08.018

Leal, S.L., Noche, J.A., Murray, E.A., Yassa, M.A., 2016. Positivity effect specific to older adults with subclinical memory impairment. Learn. Mem. Cold Spring Harb. N 23, 415421. doi:10.1101/lm.042010.116 
LeDoux, J., 2012. Rethinking the emotional brain. Neuron 73, 653-676. doi:10.1016/j.neuron.2012.02.004

LeDoux, J.E., 2014. Coming to terms with fear. Proc. Natl. Acad. Sci. 111, 2871-2878. doi:10.1073/pnas.1400335111

LeDoux, J.E., Pine, D.S., 2016. Using neuroscience to help understand fear and anxiety: A twosystem framework. Am. J. Psychiatry appi.ajp.2016.16030353. doi:10.1176/appi.ajp.2016.16030353

Lee, T.-H., Sakaki, M., Cheng, R., Velasco, R., Mather, M., 2014. Emotional arousal amplifies the effects of biased competition in the brain. Soc. Cogn. Affect. Neurosci. 9, 2067-2077. doi:10.1093/scan/nsu015

Levine, L.J., Edelstein, R.S., 2009. Emotion and memory narrowing: A review and goalrelevance approach. Cogn. Emot. 23, 833-875. doi:10.1080/02699930902738863

Levonian, E., 1972. Retention over time in relation to arousal during learning: An explanation of discrepant results. Acta Psychol. (Amst.) 36, 290-321.

Libkuman, T., Stabler, C., Otani, H., 2004. Arousal, valence, and memory for detail. Memory 12, 237-247. doi:10.1080/09658210244000630

Libkuman, T.M., Otani, H., Kern, R., Viger, S.G., Novak, N., 2007. Multidimensional normative ratings for the International Affective Picture System. Behav. Res. Methods 39, 326-334. doi:10.3758/BF03193164

Loftus, E.F., Loftus, G.R., Messo, J., 1987. Some facts about “weapon focus.” Law Hum. Behav. 11, 55-62. doi:10.1007/BF01044839

Lonergan, M., H., Olivera-Figueroa, L., A., Pitman, R., K., Brunet, A., 2013. Propranolol's effects on the consolidation and reconsolidation of long-term emotional memory in healthy participants: a meta-analysis. J. Psychiatry Neurosci. 38, 222-231.

Long, N.M., Danoff, M.S., Kahana, M.J., 2015. Recall dynamics reveal the retrieval of emotional context. Psychon. Bull. Rev. 22, 1328-1333. doi:10.3758/s13423-014-0791-2

Mackay, D.G., Shafto, M., Taylor, J.K., Marian, D.E., Abrams, L., Dyer, J.R., 2004. Relations between emotion, memory, and attention: Evidence from taboo Stroop, lexical decision, and immediate memory tasks. Mem. Cognit. 32, 474-488.

Madan, C.R., Caplan, J.B., Lau, C.S.M., Fujiwara, E., 2012. Emotional arousal does not enhance association-memory. J. Mem. Lang. 66, 695-716. doi:10.1016/j.jml.2012.04.001

Madan, C.R., Shafer, A.T., Chan, M., Singhal, A., 2016. Shock and awe: Distinct effects of taboo words on lexical decision and free recall. Q. J. Exp. Psychol. 1-18. doi:10.1080/17470218.2016.1167925

Manning, J.R., Kahana, M.J., 2012. Interpreting semantic clustering effects in free recall. Mem. Hove Engl. 20, 511-517. doi:10.1080/09658211.2012.683010

Maquet, P., Péters, J., Aerts, J., Delfiore, G., Degueldre, C., Luxen, A., Franck, G., 1996. Functional neuroanatomy of human rapid-eye-movement sleep and dreaming. Nature 383, 163-166. doi:10.1038/383163a0

Marchewka, A., Wypych, M., Michałowski, J.M., Sińczuk, M., Wordecha, M., Jednoróg, K., Nowicka, A., 2016. What Is the Effect of Basic Emotions on Directed Forgetting? Investigating the Role of Basic Emotions in Memory. Front. Hum. Neurosci. 10. doi:10.3389/fnhum.2016.00378

Marchewka, A., Żurawski, Ł., Jednoróg, K., Grabowska, A., 2014. The Nencki Affective Picture System (NAPS): Introduction to a novel, standardized, wide-range, high-quality, realistic picture database. Behav. Res. Methods 46, 596-610. doi:10.3758/s13428-013-0379-1 
Markowitsch, H.J., Calabrese, P., Wurker, M., Durwen, H., F., Kessler, J., Babinsky, R., Brechtelsbauer, D., Heuser, L., Gehlen, W., 1994. The amygdala's contribution to memory-a study on two patients with Urbach-Wiethe disease. NeuroReport 5, 13491352.

Mather, M., 2007. Emotional arousal and memory binding: An object-based framework. Perspect. Psychol. Sci. 2, 33-52.

Mather, M., Clewett, D., Sakaki, M., Harley, C.W., 2015. Norepinephrine ignites local hot spots of neuronal excitation: How arousal amplifies selectivity in perception and memory. Behav. Brain Sci. 1-100. doi:10.1017/S0140525X15000667

Mather, M., Sutherland, M.R., 2011. Arousal-Biased Competition in Perception and Memory. Perspect. Psychol. Sci. 6, 114-133. doi:10.1177/1745691611400234

Mathewson, K.J., Arnell, K.M., Mansfield, C.A., 2008. Capturing and holding attention: The impact of emotional words in rapid serial visual presentation. Mem. Cognit. 36, 182-200. doi:10.3758/MC.36.1.182

Matlin, M.W., Stang, D.J., 1978. The Pollyanna principle: selectivity in language, memory, and thought. Schenkman PubCo, Cambridge, Mass.

Matthews, W.J., Buratto, L.G., Lamberts, K., 2010. Exploring the memory advantage for moving scenes. Vis. Cogn. 18, 1393-1419. doi:10.1080/13506285.2010.492706

McCullough, A.M., Yonelinas, A.P., 2013. Cold-pressor stress after learning enhances familiarity-based recognition memory in men. Neurobiol. Learn. Mem. 106, 11-17. doi:10.1016/j.nlm.2013.06.011

McDaniel, M.A., Dornburg, C.C., Guynn, M.J., 2005. Disentangling encoding versus retrieval explanations of the bizarreness effect: Implications for distinctiveness. Mem. Cognit. 33, 270-279. doi:10.3758/BF03195316

McGaugh, J.L., 2004. The amygdala modulates the consolidation of memories of emotionally arousing experiences. Annu. Rev. Neurosci. 27, 1-28.

doi:10.1146/annurev.neuro.27.070203.144157

McGaugh, J.L., 2000. Memory--a Century of Consolidation. Science 287, 248-251. doi: $10.1126 /$ science. 287.5451 .248

McGaugh, J.L., Roozendaal, B., 2002. Role of adrenal stress hormones in forming lasting memories in the brain. Curr. Opin. Neurobiol. 12, 205-210.

McIntyre, C.K., Hatfield, T., McGaugh, J.L., 2002. Amygdala norepinephrine levels after training predict inhibitory avoidance retention performance in rats: Amygdala norepinephrine predicts long-term memory. Eur. J. Neurosci. 16, 1223-1226. doi:10.1046/j.1460-9568.2002.02188.x

McKenna, F.P., Sharma, D., 2004. Reversing the emotional Stroop effect reveals that it is not what it seems: the role of fast and slow components. J. Exp. Psychol. Learn. Mem. Cogn. 30, 382-392. doi:10.1037/0278-7393.30.2.382

McKinnon, M.C., Palombo, D.J., Nazarov, A., Kumar, N., Khuu, W., Levine, B., 2015. Threat of death and autobiographical memory: a study of passengers from Flight AT236. Clin. Psychol. Sci. J. Assoc. Psychol. Sci. 3, 487-502. doi:10.1177/2167702614542280

Mickley, K.R., Kensinger, E.A., 2008. Emotional valence influences the neural correlates associated with remembering and knowing. Cogn. Affect. Behav. Neurosci. 8, 143-152. doi:10.3758/CABN.8.2.143 
Mickley Steinmetz, K.R., Addis, D.R., Kensinger, E.A., 2010. The effect of arousal on the emotional memory network depends on valence. NeuroImage 53, 318-324. doi:10.1016/j.neuroimage.2010.06.015

Mickley Steinmetz, K.R., Kensinger, E.A., 2013. The emotion-induced memory trade-off: More than an effect of overt attention? Mem. Cognit. 41, 69-81. doi:10.3758/s13421-0120247-8

Mickley Steinmetz, K.R., Kensinger, E.A., 2009. The effects of valence and arousal on the neural activity leading to subsequent memory. Psychophysiology 46, 1190-1199. doi:10.1111/j.1469-8986.2009.00868.x

Mitsushima, D., Ishihara, K., Sano, A., Kessels, H.W., Takahashi, T., 2011. Contextual learning requires synaptic AMPA receptor delivery in the hippocampus. Proc. Natl. Acad. Sci. 108, 12503-12508. doi:10.1073/pnas.1104558108

Mogg, K., Bradley, B.P., 1999. Orienting of Attention to Threatening Facial Expressions Presented under Conditions of Restricted Awareness. Cogn. Emot. 13, 713-740. doi:10.1080/026999399379050

Montagrin, A., Brosch, T., Sander, D., 2013. Goal conduciveness as a key determinant of memory facilitation. Emot. Wash. DC 13, 622-628. doi:10.1037/a0033066

Moritz, S., Gläscher, J., Brassen, S., 2005. Investigation of mood-congruent false and true memory recognition in depression. Depress. Anxiety 21, 9-17. doi:10.1002/da.20054

Moscovitch, M., Cabeza, R., Winocur, G., Nadel, L., 2016. Episodic Memory and Beyond: The Hippocampus and Neocortex in Transformation. Annu. Rev. Psychol. 67, 105-134. doi:10.1146/annurev-psych-113011-143733

Murray, B., Kensinger, E., 2013. A review of the neural and behavioral consequences for unitizing emotional and neutral information. Front. Behav. Neurosci. 7, 42. doi:10.3389/fnbeh.2013.00042

Murty, V.P., Ritchey, M., Adcock, R.A., LaBar, K.S., 2010. fMRI studies of successful emotional memory encoding: A quantitative meta-analysis. Neuropsychologia 48, 34593469. doi:10.1016/j.neuropsychologia.2010.07.030

N'Diaye, K., Sander, D., Vuilleumier, P., 2009. Self-relevance processing in the human amygdala: gaze direction, facial expression, and emotion intensity. Emot. Wash. DC 9, 798-806. doi:10.1037/a0017845

Neiss, R., 1988. Reconceptualizing arousal: Psychobiological states in motor performance. Psychol. Bull. 103, 345-366.

Nielsen, S.E., Ahmed, I., Cahill, L., 2013a. Sex and menstrual cycle phase at encoding influence emotional memory for gist and detail. Neurobiol. Learn. Mem. 106, 56-65. doi:10.1016/j.nlm.2013.07.015

Nielsen, S.E., Ertman, N., Lakhani, Y.S., Cahill, L., 2011. Hormonal contraception usage is associated with altered memory for an emotional story. Neurobiol. Learn. Mem. 96, 378384. doi:10.1016/j.nlm.2011.06.013

Nielsen, S.E., Segal, S.K., Worden, I.V., Yim, I.S., Cahill, L., 2013b. Hormonal contraception use alters stress responses and emotional memory. Biol. Psychol. 92, 257-266. doi:10.1016/j.biopsycho.2012.10.007

Nielson, K.A., Powless, M., 2007. Positive and negative sources of emotional arousal enhance long-term word-list retention when induced as long as 30min after learning. Neurobiol. Learn. Mem. 88, 40-47. doi:10.1016/j.nlm.2007.03.005 
Nishida, M., Pearsall, J., Buckner, R.L., Walker, M.P., 2009. REM sleep, prefrontal theta, and the consolidation of human emotional memory. Cereb. Cortex N. Y. N 1991 19, 1158 1166. doi:10.1093/cercor/bhn155

Ochsner, K.N., 2000. Are affective events richly recollected or simply familiar? The experience and process of recognizing feelings past. J. Exp. Psychol. Gen. 129, 242-261. doi:10.1037//0096-3445.129.2.242

Öhman, A., Flykt, A., Esteves, F., 2001. Emotion drives attention: detecting the snake in the grass. J. Exp. Psychol. Gen. 130, 466.

Öhman, A., Mineka, S., 2001. Fears, phobias, and preparedness: Toward an evolved module of fear and fear learning. Psychol. Rev. 108, 483-522. doi:10.1037//0033-295X.108.3.483

Okuda, S., Roozendaal, B., McGaugh, J.L., 2004. Glucocorticoid effects on object recognition memory require training-associated emotional arousal. Proc. Natl. Acad. Sci. U. S. A. 101, 853-858. doi:10.1073/pnas.0307803100

O’Reilly, R.C., Bhattacharyya, R., Howard, M.D., Ketz, N., 2014. Complementary learning systems. Cogn. Sci. 38, 1229-1248. doi:10.1111/j.1551-6709.2011.01214.X

Ortony, A., Turner, T.J., 1990. What's basic about basic emotions? 97, 315-331.

Pace-Schott, E.F., Shepherd, E., Spencer, R.M.C., Marcello, M., Tucker, M., Propper, R.E., Stickgold, R., 2011a. Napping promotes inter-session habituation to emotional stimuli. Neurobiol. Learn. Mem. 95, 24-36. doi:10.1016/j.nlm.2010.10.006

Pace-Schott, E.F., Shepherd, E., Spencer, R.M.C., Marcello, M., Tucker, M., Propper, R.E., Stickgold, R., 2011b. Napping promotes inter-session habituation to emotional stimuli (English). Neurobiol Learn Mem Print 95, 24-36.

Pace-Schott, E.F., Spencer, R.M.C., Vijayakumar, S., Ahmed, N.A.K., Verga, P.W., Orr, S.P., Pitman, R.K., Milad, M.R., 2013. Extinction of conditioned fear is better learned and recalled in the morning than in the evening (English). J Psychiatr Res 47, 1776-1784.

Packard, M.G., Teather, L., A., 1998. Amygdala modulation of multiple memory systems: Hippocampus and caudate-putamen. Neurobiol. Learn. Mem. 69, 163-203.

Panksepp, J., 2005. Affective consciousness: Core emotional feelings in animals and humans. Conscious. Cogn. 14, 30-80. doi:10.1016/j.concog.2004.10.004

Panksepp, J., 1998. The periconscious substrates of consciousness: Affective states and the evolutionary origins of the self. J. Conscious. Stud. 5, 566-582.

Paré, D., 2003. Role of the basolateral amygdala in memory consolidation. Prog. Neurobiol. 70, 409-420.

Park, C.R., Zoladz, P.R., Conrad, C.D., Fleshner, M., Diamond, D.M., 2008. Acute predator stress impairs the consolidation and retrieval of hippocampus-dependent memory in male and female rats. Learn. Mem. Cold Spring Harb. N 15, 271-280. doi:10.1101/lm.721108

Park, J., 2005. Effect of arousal and retention delay on memory: a meta-analysis. Psychol. Rep. 97, 339-355.

Parkhurst, D., Law, K., Niebur, E., 2002. Modeling the role of salience in the allocation of overt visual attention. Vision Res. 42, 107-123. doi:10.1016/S0042-6989(01)00250-4

Pause, B.M., Zlomuzica, A., Kinugawa, K., Mariani, J., Pietrowsky, R., Dere, E., 2013. Perspectives on episodic-like and episodic memory. Front. Behav. Neurosci. 7, 33. doi:10.3389/fnbeh.2013.00033

Payne, J.D., Kensinger, E.A., 2011. Sleep leads to changes in the emotional memory trace: evidence from FMRI. J. Cogn. Neurosci. 23, 1285-1297. doi:10.1162/jocn.2010.21526 
Payne, J.D., Stickgold, R., Swanberg, K., Kensinger, E.A., 2008. Sleep preferentially enhances memory for emotional components of scenes. Psychol. Sci. 19, 781-788. doi:10.1111/j.1467-9280.2008.02157.x

Pessoa, L., 2013. The cognitive-emotional brain: From interactions to integration. MIT Press.

Phelps, E., 2004. Human emotion and memory: interactions of the amygdala and hippocampal complex. Curr. Opin. Neurobiol. 14, 198-202. doi:10.1016/j.conb.2004.03.015

Phelps, E.A., 2012. Emotion's impact on memory, in: Nadel, L., Sinnott-Armstrong, W.P. (Eds.), Memory and Law. Oxford University Press.

Phelps, E.A., LaBar, K.S., Anderson, A.K., O’connor, K.J., Fulbright, R.K., Spencer, D.D., 1998. Specifying the contributions of the human amygdala to emotional memory: A case study. Neurocase 4, 527-540.

Phelps, E.A., LeDoux, J.E., 2005. Contributions of the Amygdala to Emotion Processing: From Animal Models to Human Behavior. Neuron 48, 175-187. doi:10.1016/j.neuron.2005.09.025

Phelps, E.A., Ling, S., Carrasco, M., 2006. Emotion Facilitates Perception and Potentiates the Perceptual Benefits of Attention. Psychol. Sci. 17, 292-299. doi:10.1111/j.14679280.2006.01701.x

Phelps, E.A., O’Connor, K.J., Gatenby, J.C., Gore, J.C., Grillon, C., Davis, M., 2001. Activation of the left amygdala to a cognitive representation of fear. Nat. Neurosci. 4, 437-441. doi: $10.1038 / 86110$

Philippi, N., Botzung, A., Noblet, V., Rousseau, F., Després, O., Cretin, B., Kremer, S., Blanc, F., Manning, L., 2015. Impaired emotional autobiographical memory associated with right amygdalar-hippocampal atrophy in Alzheimer's disease patients. Front. Aging Neurosci. 7. doi:10.3389/fnagi.2015.00021

Pierce, B.H., Kensinger, E.A., 2011. Effects of emotion on associative recognition: valence and retention interval matter. Emot. Wash. DC 11, 139-144. doi:10.1037/a0021287

Pilcher, J.J., Huffcutt, A.I., 1996. Effects of sleep deprivation on performance: a meta-analysis. Sleep 19, 318-326.

Polyn, S.M., Erlikhman, G., Kahana, M.J., 2011. Semantic cuing and the scale insensitivity of recency and contiguity. J. Exp. Psychol. Learn. Mem. Cogn. 37, 766-775. doi:10.1037/a0022475

Polyn, S.M., Norman, K.A., Kahana, M.J., 2009. A context maintenance and retrieval model of organizational processes in free recall. Psychol. Rev. 116, 129-156. doi: $10.1037 / \mathrm{a} 0014420$

Pool, E., Brosch, T., Delplanque, S., Sander, D., 2016. Attentional bias for positive emotional stimuli: A meta-analytic investigation. Psychol. Bull. 142, 79-106.

Pourtois, G., Schettino, A., Vuilleumier, P., 2013. Brain mechanisms for emotional influences on perception and attention: What is magic and what is not. Biol. Psychol. 92, 492-512. doi:10.1016/j.biopsycho.2012.02.007

Pourtois, G., Vuilleumier, P., 2006. Dynamics of emotional effects on spatial attention in the human visual cortex. Prog. Brain Res. 156, 67-91.

Pratto, F., John, O.P., 1991. Automatic vigilance: the attention-grabbing power of negative social information. J. Pers. Soc. Psychol. 61, 380-391.

Rauchs, G., Desgranges, B., Foret, J., Eustache, F., 2005. The relationships between memory systems and sleep stages. J. Sleep Res. 14, 123-140. doi:10.1111/j.1365-

2869.2005.00450.x 
Reed, M.A., Derryberry, D., 1995. Temperament and attention to positive and negative trait information. Personal. Individ. Differ. 18, 135-147. doi:10.1016/0191-8869(94)00121-8

Reisberg, D., Heuer, F., 2003. Memory for emotional events, in: Reisberg, D., Hertel, P. (Eds.), Memory and Emotion. Oxford University Press.

Ribeiro, A.M., Barbosa, F.F., Munguba, H., Costa, M.S.M.O., Cavalcante, J.S., Silva, R.H., 2011. Basolateral amygdala inactivation impairs learned (but not innate) fear response in rats. Neurobiol. Learn. Mem. 95, 433-440. doi:10.1016/j.nlm.2011.02.004

Richard, W., Skowronski, J.J., Thompson, C.P., 2003. Life is pleasant--and memory helps to keep it that way! Rev. Gen. Psychol. 7, 203-210. doi:10.1037/1089-2680.7.2.203

Richter-Levin, G., Akirav, I., 2003. Emotional tagging of memory formation - in the search for neural mechanisms. Brain Res. Rev. 43, 247-256. doi:10.1016/j.brainresrev.2003.08.005

Riggs, L., McQuiggan, D.A., Farb, N., Anderson, A.K., Ryan, J.D., 2011. The role of overt attention in emotion-modulated memory. Emotion 11, 776-785. doi:10.1037/a0022591

Ritchey, M., Dolcos, F., Cabeza, R., 2008. Role of Amygdala Connectivity in the Persistence of Emotional Memories Over Time: An Event-Related fMRI Investigation. Cereb. Cortex 18, 2494-2504. doi:10.1093/cercor/bhm262

Ritchie, T.D., Skowronski, J.J., Wood, S.E., Walker, W.R., Vogl, R.J., Gibbons, J.A., 2006. Event self-importance, event rehearsal, and the fading affect bias in autobiographical memory. Self Identity 5, 172-195. doi:10.1080/15298860600591222

Roediger, H.L., Dudai, Y., Fitzpatrick, S.M., 2007. Science of Memory, 1st ed. Oxford University Press, Oxford, England.

Roediger, H.L., McDermott, K.B., 1995. Creating false memories: Remembering words not presented in lists. J. Exp. Psychol. Learn. Mem. Cogn. 21, 803-814.

Rolls, E., T., 2005. Emotion Explained. Oxford University Press, USA.

Roozendaal, B., 2002. Stress and memory: opposing effects of glucocorticoids on memory consolidation and memory retrieval. Neurobiol. Learn. Mem. 78, 578-595.

Roozendaal, B., 2000. Glucocorticoids and the regulation of memory consolidation. Psychoneuroendocrinology 25, 213-238. doi:10.1016/S0306-4530(99)00058-X

Roozendaal, B., Barsegyan, A., Lee, S., 2008. Adrenal stress hormones, amygdala activation, and memory for emotionally arousing experiences. Prog. Brain Res. 167, 79-97. doi:10.1016/S0079-6123(07)67006-X

Roozendaal, B., McGaugh, J.L., 2011. Memory modulation. Behav. Neurosci. 125, 797-824. doi: $10.1037 / \mathrm{a} 0026187$

Roozendaal, B., Okuda, S., Van der Zee, E.A., McGaugh, J.L., 2006. Glucocorticoid enhancement of memory requires arousal-induced noradrenergic activation in the basolateral amygdala. Proc. Natl. Acad. Sci. 103, 6741-6746.

Roseman, I.J., 2011. Emotional Behaviors, Emotivational Goals, Emotion Strategies: Multiple Levels of Organization Integrate Variable and Consistent Responses. Emot. Rev. 3, 434443. doi:10.1177/1754073911410744

Rosenthal, R., 1991. Meta-Analytic Procedures for Social Research, Rev. ed. Sage, Newbury Park, CA.

Ruci, L., Tomes, J.L., Zelenski, J.M., 2009. Mood-congruent false memories in the DRM paradigm. Cogn. Emot. 23, 1153-1165. doi:10.1080/02699930802355420

Russell, J.A., 2003. Core affect and the psychological construction of emotion. Psychol. Rev. $110,145-172$. 
Sakaki, M., Mather, M., 2012. How reward and emotional stimuli induce different reactions across the menstrual cycle. Soc. Personal. Psychol. Compass 6, 1-17. doi:10.1111/j.17519004.2011.00415.x

Sander, D., Grafman, J., Zalla, T., 2003. The human amygdala: an evolved system for relevance detection. Rev. Neurosci. 14, 303-316.

Sander, D., Grandjean, D., Scherer, K.R., 2005. A systems approach to appraisal mechanisms in emotion. Neural Netw. Off. J. Int. Neural Netw. Soc. 18, 317-352. doi:10.1016/j.neunet.2005.03.001

Schaefer, A., Nils, F., Sanchez, X., Philippot, P., 2010. Assessing the effectiveness of a large database of emotion-eliciting films: A new tool for emotion researchers. Cogn. Emot. 24, 1153-1172. doi:10.1080/02699930903274322

Schmidt, S.R., 2002. Outstanding memories: The positive and negative effects of nudes on memory. J. Exp. Psychol. Learn. Mem. Cogn. 28, 353-361. doi:10.1037//02787393.28.2.353

Schmidt, S.R., 1991. Can we have a distinctive theory of memory? Mem. Cognit. 19, 523-542.

Schmidt, S.R., Saari, B., 2007. The emotional memory effect: Differential processing or item distinctiveness? Mem. Cognit. 35, 1905-1916.

Schmidt, S.R., Schmidt, C.R., 2016. The emotional carryover effect in memory for words. Memory 24, 916-938. doi:10.1080/09658211.2015.1059859

Schultz, W., Dayan, P., Montague, P.R., 1997. A neural substrate of prediction and reward. Science 275, 1593-1599.

Schupp, H.T., Flaisch, T., Stockburger, J., Junghöfer, M., 2006. Emotion and attention: eventrelated brain potential studies. Prog. Brain Res. 156, 31-51. doi:10.1016/S00796123(06)56002-9

Schwarze, U., Bingel, U., Sommer, T., 2012. Event-Related Nociceptive Arousal Enhances Memory Consolidation for Neutral Scenes. J. Neurosci. 32, 1481-1487. doi:10.1523/JNEUROSCI.4497-11.2012

Segal, S.K., Cahill, L., 2009. Endogenous noradrenergic activation and memory for emotional material in men and women. Psychoneuroendocrinology 34, 1263-1271. doi:10.1016/j.psyneuen.2009.04.020

Sergerie, K., Lepage, M., Armony, J.L., 2005. A face to remember: emotional expression modulates prefrontal activity during memory formation. NeuroImage 24, 580-585. doi:10.1016/j.neuroimage.2004.08.051

Sharot, T., Delgado, M.R., Phelps, E.A., 2004. How emotion enhances the feeling of remembering. Nat. Neurosci. 7, 1376-1380.

Sharot, T., Phelps, E.A., 2004. How arousal modulates memory: Disentangling the effects of attention and retention. Cogn. Affect. Behav. Neurosci. 4, 294-306.

Sharot, T., Verfaellie, M., Yonelinas, A.P., 2007. How Emotion Strengthens the Recollective Experience: A Time-Dependent Hippocampal Process. PLoS ONE 2, e1068. doi:10.1371/journal.pone.0001068

Sharot, T., Yonelinas, A.P., 2008. Differential time-dependent effects of emotion on recollective experience and memory for contextual information. Cognition 106, 538-547. doi:10.1016/j.cognition.2007.03.002

Siebert, M., Markowitsch, H.J., Bartel, P., 2003. Amygdala, affect and cognition: evidence from 10 patients with Urbach-Wiethe disease. Brain J. Neurol. 126, 2627-2637. doi:10.1093/brain/awg271 
Simons, R.F., Detenber, B.H., Cuthbert, B.N., Schwartz, D.D., Reiss, J.E., 2003. Attention to Television: Alpha Power and Its Relationship to Image Motion and Emotional Content. Media Psychol. 5, 283-301.

Skinner, E.I., Fernandes, M.A., 2007. Neural correlates of recollection and familiarity: a review of neuroimaging and patient data. Neuropsychologia 45, 2163-2179. doi:10.1016/j.neuropsychologia.2007.03.007

Smith, A.P.R., Stephan, K.E., Rugg, M.D., Dolan, R.J., 2006. Task and content modulate amygdala-hippocampal connectivity in emotional retrieval. Neuron 49, 631-638. doi:10.1016/j.neuron.2005.12.025

Sommer, T., Glascher, J., Moritz, S., Buchel, C., 2008. Emotional enhancement effect of memory: Removing the influence of cognitive factors. Learn. Mem. 15, 569-573. doi:10.1101/lm.995108

Squire, L.R., Wixted, J.T., 2016. Remembering, in: Groes, S. (Ed.), Memory in the Twenty-First Century. Palgrave Macmillan UK, pp. 251-262.

Squire, L.R., Wixted, J.T., Clark, R.E., 2007. Recognition memory and the medial temporal lobe: a new perspective. Nat. Rev. Neurosci. 8, 872-883. doi:10.1038/nrn2154

Stadler, M., Roediger, H., McDermott, K., 1999. Norms for word lists that create false memories. Mem. Cognit. 27, 494-500. doi:10.3758/BF03211543

Stea, J.N., Lee, S.M., Sears, C.R., 2013. Enhancement of False Memory for Negative Material in Dysphoria: Mood Congruency or Response Bias? Cogn. Ther. Res. 37, 1189-1200. doi:10.1007/s10608-013-9557-9

Sterpenich, V., Albouy, G., Boly, M., Vandewalle, G., Darsaud, A., Balteau, E., Dang-Vu, T.T., Desseilles, M., D’Argembeau, A., Gais, S., Rauchs, G., Schabus, M., Degueldre, C., Luxen, A., Collette, F., Maquet, P., 2007. Sleep-related hippocampo-cortical interplay during emotional memory recollection. PLoS Biol. 5, e282. doi:10.1371/journal.pbio.0050282

Sterpenich, V., Albouy, G., Darsaud, A., Schmidt, C., Vandewalle, G., Dang Vu, T.T., Desseilles, M., Phillips, C., Degueldre, C., Balteau, E., Collette, F., Luxen, A., Maquet, P., 2009. Sleep promotes the neural reorganization of remote emotional memory. J. Neurosci. Off. J. Soc. Neurosci. 29, 5143-5152. doi:10.1523/JNEUROSCI.0561-09.2009

Steyvers, M., Griffiths, T.L., 2008. Rational analysis as a link between human memory and information retrieval. Probabilistic Mind Prospects Bayesian Cogn. Sci. 329-349.

Strange, B.A., Hurlemann, R., Dolan, R.J., 2003. An emotion-induced retrograde amnesia in humans is amygdala-and $\beta$-adrenergic-dependent. Proc. Natl. Acad. Sci. 100, 1362613631.

Sutherland, M.R., Mather, M., 2012. Negative arousal amplifies the effects of saliency in shortterm memory. Emotion 12, 1367-1372. doi:10.1037/a0027860

Talarico, J.M., Berntsen, D., Rubin, D.C., 2009. Positive emotions enhance recall of peripheral details. Cogn. Emot. 23, 380-398. doi:10.1080/02699930801993999

Talarico, J.M., LaBar, K.S., Rubin, D.C., 2004. Emotional intensity predicts autobiographical memory experience. Mem. Cognit. 32, 1118-1132. doi:10.3758/BF03196886

Talmi, D., 2013. Enhanced Emotional Memory: Cognitive and Neural Mechanisms. Curr. Dir. Psychol. Sci. 22, 430-436. doi:10.1177/0963721413498893

Talmi, D., Luk, B.T.C., McGarry, L.M., Moscovitch, M., 2007a. The contribution of relatedness and distinctiveness to emotionally-enhanced memory. J. Mem. Lang. 56, 555-574.

doi:10.1016/j.jml.2007.01.002 
Talmi, D., McGarry, L.M., 2012. Accounting for immediate emotional memory enhancement. J. Mem. Lang. 66, 93-108. doi:10.1016/j.jml.2011.07.009

Talmi, D., Moscovitch, M., 2004. Can semantic relatedness explain the enhancement of memory for emotional words? Mem. Cognit. 32, 742-751.

Talmi, D., Schimmack, U., Paterson, T., Moscovitch, M., 2007b. The role of attention and relatedness in emotionally enhanced memory. Emotion 7, 89-102. doi:10.1037/15283542.7.1.89

Talmi, D., Ziegler, M., Hawksworth, J., Lalani, S., Herman, C.P., Moscovitch, M., 2013. Emotional stimuli exert parallel effects on attention and memory. Cogn. Emot. 27, 530538. doi:10.1080/02699931.2012.722527

Tanguay, A.N., Ramdeen, K.T., Muyingo, L., Beaudoin, C., Davidson, P.S.R., 2016. A collection of unfamiliar emotional movie clips, in: Proceedings of the 23rd Annual Meeting of the Cognitive Neuroscience Society. New York City, USA.

Teyler, T.J., Rudy, J.W., 2007. The hippocampal indexing theory and episodic memory: Updating the index. Hippocampus 17, 1158-1169. doi:10.1002/hipo.20350

Touryan, S.R., Marian, D.E., Shimamura, A.P., 2007. Effect of negative emotional pictures on associative memory for peripheral information. Mem. Hove Engl. 15, 154-166. doi:10.1080/09658210601151310

Trammell, J.P., Clore, G.L., 2014. Does Stress Enhance or Impair Memory Consolidation? Cogn. Emot. 28, 361-374. doi:10.1080/02699931.2013.822346

Tsuno, N., Besset, A., Ritchie, K., 2005. Sleep and depression. J. Clin. Psychiatry 66, 12541269.

Tulving, E., 1985. Elements of Episodic Memory. Clarendon Press, New York, NY.

Turner, T.J., Ortony, A., 1992. Basic emotions: can conflicting criteria converge? Psychol. Rev. 99, 566-571.

Unkelbach, C., Fiedler, K., Bayer, M., Stegmüller, M., Danner, D., 2008. Why positive information is processed faster: The density hypothesis. J. Pers. Soc. Psychol. 95, 36-49. doi:10.1037/0022-3514.95.1.36

van der Helm, E., Gujar, N., Nishida, M., Walker, M.P., 2011. Sleep-dependent facilitation of episodic memory details. PloS One 6, e27421. doi:10.1371/journal.pone.0027421

van Stegeren, A.H., 2008. The role of the noradrenergic system in emotional memory. Acta Psychol. (Amst.) 127, 532-541. doi:10.1016/j.actpsy.2007.10.004

van Stegeren, A.H., Goekoop, R., Everaerd, W., Scheltens, P., Barkhof, F., Kuijer, J.P.A., Rombouts, S.A.R.B., 2005. Noradrenaline mediates amygdala activation in men and women during encoding of emotional material. NeuroImage 24, 898-909. doi:10.1016/j.neuroimage.2004.09.011

Vuilleumier, P., Armony, J.L., Driver, J., Dolan, R.J., 2003. Distinct spatial frequency sensitivities for processing faces and emotional expressions. Nat. Neurosci. 6, 624-631. doi:10.1038/nn1057

Vuilleumier, P., Driver, J., 2007. Modulation of visual processing by attention and emotion: windows on causal interactions between human brain regions. Philos. Trans. R. Soc. B Biol. Sci. 362, 837-855. doi:10.1098/rstb.2007.2092

Wagner, U., Born, J., 2008. Memory consolidation during sleep: interactive effects of sleep stages and HPA regulation. Stress Amst. Neth. 11, 28-41.

doi:10.1080/10253890701408822 
Wagner, U., Fischer, S., Born, J., 2002a. Changes in emotional responses to aversive pictures across periods rich in slow-wave sleep versus rapid eye movement sleep. Psychosom. Med. 64, 627-634.

Wagner, U., Fischer, S., Born, J., 2002b. Changes in emotional responses to aversive pictures across periods rich in slow-wave sleep versus rapid eye movement sleep. Psychosom. Med. 64, 627-634. doi:10.1097/01.PSY.0000021940.35402.51

Wagner, U., Gais, S., Born, J., 2001. Emotional memory formation is enhanced across sleep intervals with high amounts of rapid eye movement sleep. Learn. Mem. Cold Spring Harb. N 8, 112-119. doi:10.1101/lm.36801

Wagner, U., Hallschmid, M., Rasch, B., Born, J., 2006. Brief sleep after learning keeps emotional memories alive for years. Biol. Psychiatry 60, 788-790. doi:10.1016/j.biopsych.2006.03.061

Walker, M.P., van der Helm, E., 2009. Overnight therapy? The role of sleep in emotional brain processing. Psychol. Bull. 135, 731-748. doi:10.1037/a0016570

Walker, W.R., Skowronski, J.J., 2009. The Fading affect bias: But what the hell is it for? Appl. Cogn. Psychol. 23, 1122-1136. doi:10.1002/acp.1614

Wheeler, M.A., Stuss, D.T., Tulving, E., 1997. Toward a theory of episodic memory: the frontal lobes and autonoetic consciousness. Psychol. Bull. 121, 331-354.

Wixted, J.T., 2004. The Psychology and Neuroscience of Forgetting. Annu. Rev. Psychol. 55, 235-269. doi:10.1146/annurev.psych.55.090902.141555

Wixted, J.T., 1990. Analyzing the empirical course of forgetting. J. Exp. Psychol. Learn. Mem. Cogn. 16, 927.

Wixted, J.T., Squire, L.R., 2010. The role of the human hippocampus in familiarity-based and recollection-based recognition memory. Behav. Brain Res. 215, 197-208. doi:10.1016/j.bbr.2010.04.020

Xu, X., Zhao, Y., Zhao, P., Yang, J., 2011. Effects of level of processing on emotional memory: Gist and details. Cogn. Emot. 25, 53-72. doi:10.1080/02699931003633805

Yerkes, R.M., Dodson, J.D., 1908. The relation of strength of stimulus to rapidity of habitformation. J. Comp. Neurol. Psychol. 18, 459-482.

Yiend, J., 2010. The effects of emotion on attention: A review of attentional processing of emotional information. Cogn. Emot. 24, 3-47. doi:10.1080/02699930903205698

Yonelinas, A.P., Parks, C.M., Koen, J.D., Jorgenson, J., Mendoza, S.P., 2011. The effects of post-encoding stress on recognition memory: Examining the impact of skydiving in young men and women. Stress 14, 136-144. doi:10.3109/10253890.2010.520376

Yonelinas, A.P., Ritchey, M., 2015. The slow forgetting of emotional episodic memories: an emotional binding account. Trends Cogn. Sci. 19, 259-267. doi:10.1016/j.tics.2015.02.009

Yoo, S.-S., Gujar, N., Hu, P., Jolesz, F.A., Walker, M.P., 2007. The human emotional brain without sleep--a prefrontal amygdala disconnect. Curr. Biol. CB 17, R877-878. doi:10.1016/j.cub.2007.08.007 